\title{
GROUPS WITH ABELIAN CENTRAL QUOTIENT GROUP $\dagger$
}

\author{
BY \\ REINHOLD BAER
}

In recent years much progress has been made in the study of groups whose central quotient groups are abelian. $\ddagger$ Such a group is an extension of an abelian group by an abelian group, and the usual method of solving the implied extension problem may be described as follows: If $G$ is such a group, then a maximum abelian subgroup $V$ of $G$ is chosen. $V$ contains the central of $G$, and $G / V$ represents therefore exactly an abelian group of automorphisms of the abelian group $V$. Thus it is possible to apply the results of the theory of automorphisms of abelian groups. This method is rather powerful and yields very interesting results. On the other hand it is not restricted to this class of groups and may in fact be applied to all groups with abelian commutator groups. $\$$ Finally it has to be mentioned that this method is not an invariant one, since the maximum abelian subgroups are in no sense uniquely determined.

In this paper another method will be indicated. If $G$ is a group whose central quotient group is abelian, then preference is given to a subgroup $S$ which is situated between the central and the commutator group of $G$. This subgroup $S$ will be left indeterminate as long as possible. But as soon as the final results are reached, either the central or the commutator group of $G$ will take the place of $S$ according to which of these choices will give better results.

The extension problem presents itself now in the following form: To characterize those groups whose central contains a given abelian group $S$ and whose quotient group $(\bmod S)$ is isomorphic to a preassigned abelian group $G^{*}$. Each group with these properties induces certain invariant relations between the given abelian groups $G^{*}$ and $S$, and these invariants turn out to be characteristic invariants, provided $G^{*}$ is a direct product of (a finite or infinite number of finite and infinite) cyclic groups. This last hypothesis will be the only restriction of generality imposed on the investigated groups, so that the finite groups with abelian central quotient groups are included in our treatment.

$\dagger$ Presented to the Society, September 7, 1937; receivod by the editors September 20, 1937.

$\ddagger$ Cf., for example, H. R. Brahana, American Journal of Mathematics, vol. 57 (1935), pp. 645667, and Duke Mathematical Journal, vol. 1 (1935), pp. 185-197; also C. Hopkins, these Transactions, vol. 37 (1935), pp. 161-195; vol. 41 (1937), pp. 287-313.

$\S$ K. Taketa, Japanese Journal of Mathematics, vol. 13 (1937), pp. 129-232. 
1. Basic concepts and formulas. If $G$ is any group, then $Z(G)$ denotes the central, and $C(G)$ the commutator group of $G$. If $n$ is any positive integer, then $G_{n}$ is generated by the elements $x$ which satisfy $x^{n}=1$, and $G^{n}$ is generated by the elements of the form $x^{n}$ for $x$ in $G$. (If $G$ is an abelian group, then $G_{n}$ consists of the elements $x$ with $x^{n}=1$, and $G^{n}$ consists of the elements $x^{n}$ for $x$ in G.) Finally let $(x, y)=x y x^{-1} y^{-1}$.

(1.1) If $G$ and $S$ are groups such that $C(G) \leqq S \leqq Z(G)$, then the operation $\left(S<G ; x^{*}, y^{*}\right)=x^{*} \circ y^{*}$, which is defined by $S x \circ S y=(x, y)$, obeys the following rules:

(1) $x^{*} \circ y^{*}$ is, for every $x^{*}, y^{*}$ in $G^{*}=G / S$, a uniquely determined element in $S$.

(2) $1=x^{*} \circ x^{*}=\left(x^{*} \circ y^{*}\right)\left(y^{*} \circ x^{*}\right)$.

(3) $x^{*} \circ y^{*} z^{*}=\left(x^{*} \circ y^{*}\right)\left(x^{*} \circ z^{*}\right)$.

(4) The element $z$ in $G$ belongs to $Z(G)$ if, and only if, $S z \circ x^{*}=1$ for every $x^{*}$ in $G^{*}$.

Proof. The statements (1), (2), and (4) are obvious. (3) may be proved as follows:

$$
\begin{aligned}
S x \circ S y S z & =x y z x^{-1} z^{-1} y^{-1}=x y x^{-1} x z x^{-1} z^{-1} y^{-1} \\
& =(x, y)(x, z),
\end{aligned}
$$

since every commutator is an element of the central.

An obvious consequence of assertion (1.1), (4) is the following proposition:

(1.2) Suppose that $C(G) \leqq S \leqq Z(G)$. Then $S=Z(G)$ if, and only if, 1 is the only element $w^{*}$ in $G / S$ such that $w^{*} \circ x^{*}=1$ for every element $x^{*}$ in $G / S$.

(1.3) If $x$ and $y$ are elements of the group $G$ with abelian central quotient group, then

$$
(x y)^{i}=(y, x)^{i(i-1) 2^{-1}} x^{i} y^{i}
$$

for every positive integer $i$.

Proof. This statement is certainly true for $i=1$. If $1<i$, then

$$
\begin{aligned}
x^{i-1} y^{i-1} x y & =x^{i} x^{-1} y^{i-1} x y^{1-i} y^{i}=x^{i}\left(x^{-1}, y^{i-1}\right) y^{i} \\
& =(y, x)^{i-1} x^{i} y^{i},
\end{aligned}
$$

since the commutators are elements of the central, and since (2) and (3) of (1.1) may be applied. If (1.3) holds true for $i-1$, then

$$
(x y)^{i}=(y, x)^{(i-1)(i-2) 2^{-1}} x^{i-1} y^{i-1} x y=(y, x)^{i(i-1) 2^{-1}} x^{i} y^{i},
$$

and thus (1.3) holds true for cvery $i$. 
(1.4) Suppose that $C(G) \leqq S \leqq Z(G)$ and that $x$ and $y$ are elements of $G$ such that $S x$ and $S y$ are both contained in $(G / S)_{n}$.

(a) If at least one of the elements $S x$ and $S y$ is contained in $(G / S)^{2}$ (this would certainly be the case, if $n$ is odd), then $(x y)^{n}=x^{n} y^{n}$.

(b) If $n=2 m$ is an even integer, then

$$
(x y)^{2 m}=(S x \circ S y)^{m} x^{2 m} y^{2 m} \text { and }\left((S x \circ S y)^{m}\right)^{2}=1 .
$$

Proof. It follows from (1.3) that

$$
(x y)^{n}=(S y \circ S x)^{n(n-1) 2^{-1}} x^{n} y^{n} ;
$$

and the statement (b) is now a consequence of (1.1). In order to prove (a), assume that $S y$ belongs to $(G . S)^{2}$. Then $S y=S z^{2}$ for some $z$ in $G$ and

$$
(S y \circ S x)^{n(n-1) 2^{-1}}=\left(S z \circ S x^{n}\right)^{n-1}=1
$$

by (1.1). This completes the proof.

(1.5) If $C(G) \leqq S \leqq Z(G)$, and if the function $P\left(n, x^{*}\right)=P\left(S<G ; n, x^{*}\right)$ is defined by $P(n, S x)=S^{n} x^{n}$, then the following statements are true:

(1) $P\left(n, x^{*}\right)$ is, for every $x^{*}$ in $(G / S)_{n}$, a uniquely determined element in $S / S^{n}$

(2') If $x^{*}$ and $y^{*}$ are both elements of $(G / S)_{n}$ and at least one of them is an element of $(G / S)^{2}$ (for example, if $n$ is odd $)$, then $P\left(n, x^{*} y^{*}\right)=P\left(n, x^{*}\right) P\left(n, y^{*}\right)$.

$\left(2^{\prime \prime}\right)$ If $x^{*}$ and $y^{*}$ are both elements of $(G / S)_{2 m}$, then

$$
P\left(2 m, x^{*} y^{*}\right)=\left(x^{*} \circ y^{*}\right)^{m} P\left(2 m, x^{*}\right) P\left(2 m, y^{*}\right),
$$

and $\left(x^{*} \circ y^{*}\right)^{m}$ is either the unit or an element of order 2 .

(3) $P\left(n m, x^{*}\right)=P\left(n, x^{*}\right)^{m}$ for positive $m$ and $x^{*}$ in $(G / S)_{n}$; and $P\left(n, x^{*_{m}}\right)$ $=S^{n} P\left(n m, x^{*}\right)$ for $x^{*}$ in $(G / S)_{n m}$.

Proof. (1), (2'), and $\left(2^{\prime \prime}\right)$ are consequences of (1.4). If $S x$ belongs to $(G / S)_{n}$ and $m$ is a positive integer, then $S x$ belongs to $(G / S)_{n m}$ and $P(n, S x)^{m}$ $=\left(S^{n} x^{n}\right)^{m}=S^{n m} x^{n m}=P(n m, S x)$. If $S x$ belongs to $(G / S)_{n m}$, then $S x^{m}$ belongs to $(G / S)_{n}$, and $P\left(n, S x^{m}\right)=S^{n} x^{n m}=S^{n} S^{n m} x^{n m}=S^{n} P(n m, S x)$.

2. Existence theorems. We prove the following theorem:

THEOREM 2.1. Suppose that $S$ and $G^{*}$ are abelian groups, that $x^{*} \circ y^{*}$ is, for every $x^{*}$ and $y^{*}$ in $G^{*}$, an element in $S$, and that $P\left(n, x^{*}\right)$ is an element in $S / S^{n}$ for every positive integer $n$ (if $n$ is such that elements of order $n$ exist in $G^{*}$ ) and for every $x^{*}$ in $G_{n}{ }^{*}$.

There exists a group $G$ such that $C(G) \leqq S \leqq Z(G)$ and an isomorphism $\gamma$ of $G / S$ upon the whole group $G^{*}$ such that $(S x)^{r} \circ(S y)^{r}=(x, y)$ for $x$ and $y$ in $G$ and $P\left(n,(S x)^{r}\right)=S^{n} x^{n}$ for $S x$ in $(G / S)_{n}$ (provided there exist elements of order $n$ in $\left.G^{*}\right)$ if, and only if, the following conditions are satisfied: 
(1) $1=x^{*} \circ x^{*}=\left(x^{*} \circ y^{*}\right)\left(y^{*} \circ x^{*}\right), x^{*} \circ y^{*} z^{*}=\left(x^{*} \circ y^{*}\right)\left(x^{*} \circ z^{*}\right)$.

(2) $P\left(n, x^{*} y^{*}\right)=P\left(n, x^{*}\right) P\left(n, y^{*}\right)$, if the $n$ is odd; and $P\left(2 m, x^{*} y^{*}\right)$ $=\left(x^{*} \circ y^{*}\right)^{m} P\left(2 m, x^{*}\right) P\left(2 m, y^{*}\right)$.

(3) $P\left(n m, x_{m}^{*}\right)=P\left(n, x^{*}\right)^{m}$, if $x^{*}$ is an element of $G_{n}^{*} . P\left(n, x^{*}\right)=S^{n} P\left(n m, x^{*}\right)$, if $x^{*}$ is an element of $G_{m n}^{*}$.

Proof. The necessity of the conditions (1) to (3) is a consequence of (1.1) and (1.5). Suppose now that conditions (1) to (3) are satisfied. There exist an ordinal number $t$ and, for every ordinal number $v$ with $0 \leqq v \leqq t$, a group $G^{*}(v)$ with the following properties: $G^{*}(0)=1, G^{*}(v)<G^{*}\left(v^{\prime}\right)$ for $v<v^{\prime}$, $G^{*}(t)=G^{*} ; G^{*}(v+1) / G^{*}(v)$ is a cyclic group whose order is either infinite or a prime number; and if $v$ is a limit ordinal, then $G^{*}(v)$ is the join of the groups $G^{*}(u)$ for $u<v$.

By complete (transfinite) induction with regard to $v$, groups $G(v)$ and isomorphisms $\gamma(v)$ will be defined which obey the following rules:

(i) $C(G(v)) \leqq S \leqq Z(G(v))$.

(ii) $G(v)<G\left(v^{\prime}\right)$ for $0 \leqq v<v^{\prime} \leqq t$.

(iii) $\gamma(v)$ is an isomorphism of $G(v) / S$ upon the whole group $G^{*}(v)$.

(iv) If $x$ is an element in $G(v)$ and $v<v^{\prime}$, then $(S x)^{\gamma(v)}=(S x)^{\gamma\left(v^{\prime}\right)}$.

(v) If $x$ and $y$ are elements in $G(v)$, then $(x, y)=(S x)^{\gamma(v)} \circ(S y)^{\gamma(v)}$.

(vi) If $G^{*}$ contains elements of the finite order $n$, and if $S x$ belongs to $(G(v) / S)_{n}$, then $S^{n} x^{n}=P\left(n,(S x)^{\gamma(v)}\right)$.

The choices $G(0)=S$ and $\gamma(0)=1$ are in accordance with these rules, since $G^{*}(0)=1$. It may therefore be assumed that for every $u<v$ a group $G(u)$ and an isomorphism $\gamma(u)$ have been defined which satisfy (i) to (vi).

Case 1. $v=w+1$ is not a limit ordinal. $G^{*}(w+1) / G^{*}(w)$ is a cyclic group. It is either infinite or its order is a prime number $p$. Denote by $b^{*}$ an element of $G^{*}(v)$ generating $G^{*}(v)\left(\bmod G^{*}(w)\right)$. If $G^{*}(w) b^{*}$ contains elements of finite order, then $b^{*}$ may be chosen as an element of minimum order in its class $G^{*}(w) b^{*}$. If $G^{*}(w+1) / G^{*}(w)$ is of finite order $p$, then let $c$ be an element in $G(w)$ such that $(S c) \gamma(w)=b^{* p}$.

In order to define $G(v)$ and $\gamma(v)$ we require first the following results:

(2.11) An automorphism $\beta$ of $G(w)$ is defined by $x^{\beta}=\left(b^{*} \circ(S x)^{\gamma(w)}\right) x$. All the elements of $S$ are fixed elements of $\beta$. If $G^{*}(v) / G^{*}(w)$ is of finite order $p$, then $c$ is a fixed element of $\beta$ and the automorphism $\psi=\beta^{p}$ is the inner automorphism of $G(w)$ induced by $c$.

By its definition $x^{\beta}$ is, for every $x$ in $G(w)$, a uniquely determined element of $G(w)$. If $x$ is an element in $S$, then $S x=S$ and $x^{\beta}=x$ by (1). If $x$ and $y$ are elements of $G(w)$, then $(x y)^{\beta}=\left(b^{*} \circ(S x y)^{\gamma(w)}\right) x y=\left(b^{*} \circ(S x)^{\gamma(w)}\right)\left(b^{*} \circ(S y)^{\gamma(w)}\right) x y$ $=x^{\beta} y^{\beta}$ by (1) and (i). If $x^{\beta}=1$, then $x$ is an element of $S$, consequently $x=1$. 
From these facts it follows that $\beta$ is a (proper) automorphism of $G(w)$.

Suppose now that $G^{*}(v) / G^{*}(w)$ is of finite order $p$. Then $c^{\beta}=\left(b^{*} \circ(S c) \gamma(w)\right) c$ $=\left(b^{*} \circ b^{* p}\right) c=c$ by (1). Since the elements of $S$ are fixed elements of the auto$\operatorname{morphism} \beta$,

$$
x^{\psi}=\left(b^{*} \circ(S x)^{\gamma(w)}\right)^{p} x=\left(b^{* p} \circ(S x)^{\gamma(w)}\right) x
$$

by (1), and

$$
x^{\psi}=\left((S c)^{\gamma(w)} \circ(S x)^{\gamma(w)}\right) x=c x c^{-1}
$$

by (v). This completes the proof of (2.11).

(2.12) There exists a group $G(v)$ with the following properties:

(a) $G(w)$ is a normal subgroup of $G(v)$, and $G(v) / G(w)$ is a cyclic group of the same order as $G^{*}(v) / G^{*}(w)$.

(b) $G(v)$ contains an element $b$ which generates $G(v)(\bmod G(w))$ and satisfies the relation $b x b^{-1}=x^{\beta}$ for every $x$ in $G(w)$.

(c) If $G^{*}(v) / G^{*}(w)$ is of finite order $p$, then $b^{p}$ is an element of $G(w)$, and $S c=S b^{p}$.

(d) If $b^{*}$ is an element of finite order $p n$, then $S^{p n} b^{p n}=P\left(p n, b^{*}\right)$.

To prove this note first that $G^{*}(v) / G^{*}(w)$ is of finite order $p$ if $b^{*}$ is of finite order. The order of $b^{*}$, if finite, is therefore a multiple $p n$ of $p$. It follows from (3) and (vi) that there exists an element $c^{\prime}$, satisfying $S c=S c^{\prime}$ and $P\left(p n, b^{*}\right)=S^{p n} c^{\prime n}$. Now it follows from (2.11) and general theorems in the theory of extensions of groups $\dagger$ that the group $G(v)$ which is generated by $G(w)$ and an element $b$ subject to the relations $b x b^{-1}=x^{\beta}$ for $x$ in $G(w)$ and $b^{p}=c$, if $b^{*}$ is of infinite order and $G^{*}(v) / G^{*}(w)$ of finite order $p$, or $b^{p}=c^{\prime}$, if $b^{*}$ is of finite order $p n$ and $G^{*}(v) / G^{*}(w)$ of finite order $p$, satisfies the conditions (a) to (d).

(2.13) There exists one and only one isomorphism $\gamma(v)$ of $G(v) / S$ upon $G^{*}(v)$ which satisfies

for $x$ in $G(w)$.

$$
(S b)^{\gamma(v)}=b^{*}, \quad(S x)^{\gamma(v)}=(S x)^{\gamma(w)}
$$

This is an obvious consequence of the following facts: $\beta$ induces in $S$ and in $G(v) / S$ the identical automorphism; if $G^{*}(v) / G^{*}(w)$ is of finite order $p$, then $b^{* p}=(S c)^{\gamma(w)}=\left(S c^{\prime}\right)^{\gamma(w)}$.

That these definitions of $G(v)$ and $\gamma(v)$ are in accordance with (i) to (iv) is clear. Any pair of elements in $G(v)$ has the form $x b^{i}, y b^{i}$ with $x, y$ in $G(w)$. Hence, by (1), (i), (v) is a consequence of

† A. M. Turing, The extension of groups, Compositio Mathematica, vol. 5 (1938), pp. 557-567. 


$$
\begin{aligned}
\left(x b^{i}, y b^{j}\right) & =x b^{i} y b^{i} b^{-i} x^{-1} b^{-j} y^{-1}=x y^{\beta^{i}}\left(x^{-1}\right)^{\beta^{i}} y^{-1} \\
& =\left(b^{* i} \circ(S y)^{\gamma(w)}\right)\left((S x)^{\gamma(w)} \circ b^{* j}\right)(x, y) \\
& =\left(S x b^{i}\right)^{\gamma(v)} \circ\left(S y b^{i}\right)^{\gamma(v)} .
\end{aligned}
$$

Suppose that $G^{*}$ contains elements of order $m$ and that $x b^{i}$ is an element in $G(v)$ such that $\left(x b^{i}\right)^{m}$ is an element of $S$. Then it follows from (i) and (1.3) that

$$
\left(x b^{i}\right)^{m}=\left((S x)^{\gamma(w)} \circ b^{*}\right)^{-i m(m-1) 2^{-1}} x^{m} b^{i m} ;
$$

consequently $x^{m} b^{i m}$ is an element of $S$. Therefore $i=0$, if $G^{*}(v) / G^{*}(w)$ is infinite, and $S^{m} x^{m}=P\left(m(S x)^{\gamma(w)}\right)$ by (vi) (which condition is satisfied in $G(w))$. If $G^{*}(v) / G^{*}(w)$ is of finite order $p$, then it may be assumed without loss in generality that $0 \leqq i<p$. If $i=0$, then the above argument may be applied; if $i \neq 0$, then $i$ and $p$ are relatively prime. Since $b^{i m}$ is an element of $G(w)$, this implies that $m$ is a multiple of $p$. Since $i$ is relatively prime to $p$, and since $b^{i}$ and $x b^{i}$ are elements of the same class $(\bmod G(w))$, it follows that the order of $b^{*_{i}}$ is finite and a divisor of the order of $\left(S x b^{i}\right)^{\gamma(v)}$. Hence $p n$ is a divisor of $m$, and $b^{i m}$, as well as $x^{m}$, is an element of $S$. Consequently

$$
S^{m}\left(x b^{i}\right)^{m}=\left((S x)^{\gamma(w)} \circ b^{*}\right)^{-i m(m-1) 2^{-1}} P\left(m,(S x)^{\gamma(w)}\right) P\left(m, b^{* i}\right)
$$

by (vi) applied on $G(w)$, by (2.12), (d) and by condition (3); and

$$
S^{m}\left(x b^{i}\right)^{m}=P\left(m,(S x)^{\gamma(w)} b^{* i}\right)=P\left(m,\left(S x b^{i}\right)^{\gamma(v)}\right)
$$

by (2) and (2.13). Thus the condition (vi) is satisfied by $G(v)$ and $\gamma(v)$.

Case 2. $v$ is a limit ordinal. Let $G(v)$ be the uniquely determined group which contains all the groups $G(u)$ for $u<v$ and is just their join, and let $\gamma(v)$ be the uniquely determined isomorphism of $G(v) / S$ upon $G^{*}(v)$ which coincides on every $G(u) / S$ for $u<v$ with $\gamma(v)$. That the conditions (i) to (vi) are satisfied is clear.

Thus $G(v), \gamma(v)$ are defined for every $0 \leqq v \leqq t$. Since $G^{*}(t)=G^{*}$, it follows that the group $G(t)=G$ and the isomorphism $\gamma(t)=\gamma$ meet all the requirements of the theorem. This completes the proof.

Corollary 2.2. Suppose that $S$ and $G^{*}$ are abelian groups and that $x^{*} \circ y^{*}$ is, for every $x^{*}$ and $y^{*}$ in $G^{*}$, an element in $S$. Then there exists a group $G$ such that $C(G) \leqq S \leqq Z(G)$ and an isomorphism $\gamma$ of $G / S$ upon the whole group $G^{*}$ such that

$$
(S x)^{\gamma} \circ(S y)^{\gamma}=(x, y)
$$

for $x$ and $y$ in $G$ if, and only if,

$$
1=x^{*} \circ x^{*}=\left(x^{*} \circ y^{*}\right)\left(y^{*} \circ x^{*}\right), \quad x^{*} \circ y^{*} z^{*}=\left(x^{*} \circ y^{*}\right)\left(x^{*} \circ z^{*}\right) \text {. }
$$


Proof. The necessity of the condition is a consequence of (1.1). Suppose now that the condition is satisfied. Denote by $F\left(G^{*}\right)$ the subgroup of all the elements of finite order in $G^{*}$. Then there exists a basis $B^{*}$ of $F\left(G^{*}\right)$ $\left(\bmod F\left(G^{*}\right)\right) \dagger$ which contains, for every positive integer $i$, a basis $B_{i}^{*}$ of $G_{2}^{*}{ }_{i}$ modulo the cross cut of $G^{* 2}$ and $G_{2}^{*}{ }^{*} . \ddagger$ ( $B_{i}$ is the cross cut of $B^{*}$ and $G_{2}^{*}$.) If $n$ is an odd integer and $m$ a non-negative integer, then every element $x^{*}$ in $G_{n 2^{m}}^{*}$ may be represented in one and only one way in the form

$$
x^{*} \equiv \prod_{b^{*} \in B_{m}} b^{* h\left(x^{*}, b^{*}\right)}\left(\bmod G^{* 2}\right),
$$

$h\left(x^{*}, b^{*}\right)=0$ or 1 , and $h\left(x^{*}, b^{*}\right)=0$ for almost every $b^{*}$. If $b_{1}^{*}, \cdots, b_{k}^{*}$ are exactly those elements $b^{*}$ in $B_{m}^{*}$ such that $h\left(x^{*}, b_{i}^{*}\right)=1$, then put

$$
P\left(n 2^{m}, x^{*}\right)=S^{n 2^{m}} \prod_{j<i}\left(b_{j}^{*} \circ b_{i}^{*}\right)^{2^{m-1}} .
$$

It is now fairly obvious that the functions $x^{*} \circ y^{*}$ and $P\left(r, x^{*}\right)$ satisfy the conditions (1) to (3) of the Theorem 2.1, and the existence of a group $G$ and of an isomorphism $\gamma$, meeting the requirements of the Corollary 2.2, is a consequence of Theorem 2.1.

COROLLARY 2.3. If $Z$ and $G$ are abelian groups, then the necessary and sufficient condition for the existence of a group whose central is $Z$ and whose central quotient group is $G$ is the existence of an operation $x$ of of the elements $x$ and $y$ in $G$ with values in $Z$, satisfying the conditions:

(1) $x \circ y$ is for every $x$ and $y$ in $G$ an element in $Z$.

(2) $1=x \circ x=(x \circ y)(y \circ x)$.

(3) $x \circ y z=(x \circ y)(x \circ z)$.

(4) $w \circ x=1$ for every $x$ in $G$ if, and only if, $w=1$.

This corollary is a consequence of Corollary 2.2 and of (1.2).

3. Factor sets. Suppose that $C(G) \leqq S \leqq Z(G)$ and that $G^{*}=G / S$ is a direct product of cyclic groups. Let $B^{*}$ be a basis of $G^{*}$, and let $B$ be some set of representatives in $G$ of the classes in $B^{*}$. The elements in $B$ may be ordered in some way. It is important to note that the following formulas depend on the way in which $B$ has been ordered.

If $x^{*}$ is any element of finite order in $G^{*}$, then let $n\left(x^{*}\right)$ be its order. Now every element $x$ in $G$ may be represented in one and only one way as

$$
x=s(x) \prod_{b \in B} b^{n(x, b)},
$$

$\dagger$ K. Taketa, Japanese Journal of Mathematics, vol. 13 (1937), pp. 129-232.

‡ Cf., for example, R. Baer, American Journal of Mathematics, vol. 59 (1937), pp. 99-117, particularly $\S 1$. 
where the factors of the product are ordered in the same way as $B$, almost every $n(x, b)=0$, where $0 \leqq n(x, b)<n(S b)$ (if $S b$ is an element of finite order), and $s(x)$ is an element in $S$.

If $x$ and $y$ are two elements in $G$, then integers $h(x, y ; b)$, which may only be 0 or 1 , are uniquely determined by the equation

$$
n(x y, b)+h(x, y ; b) n(S b)=n(x, b)+n(y, b)
$$

if $S b$ is of finite order, and

$$
n(x y, b)=n(x, b)+n(y, b)
$$

if $S b$ is of infinite order. Put furthermore

$$
a(x, y)=\prod^{\prime} b^{n(S b)},
$$

where $\Pi^{\prime}$ indicates a product taken over all those $b$ in $B$ such that $S b$ is of finite order and $h(x, y ; b)=1$; and put

$$
c(x, y)=\prod_{b<b^{\prime}}\left(S b^{\prime} \circ S b\right)^{n\left(x, b^{\prime}\right) n(y, b)},
$$

where $S b \circ S b^{\prime}=\left(b, b^{\prime}\right)$. Then it follows from (1.1) that

$$
s(x y)=s(x) s(y) a(x, y) c(x, y) .
$$

Note that $a(x, y)=a(S x, S y)=a(S y, S x)$ and $c(x, y)=c(S x, S y)$. The product $a\left(x^{*}, y^{*}\right) c\left(x^{*}, y^{*}\right)$ is, in the usual terminology, $\dagger$ a factor set of the extension $G$ of $S$ by $G^{*}$ satisfying $S \leqq Z(G)$.

4. Existence of transformations. Suppose that $S, G$ and $T, H$ are groups with the property that $C(G) \leqq S \leqq Z(G)$ and $C(H) \leqq T \leqq Z(H)$. Then $\phi$ is called an $S$-T-transformation of $G$ into $H$, if $\phi$ obeys the following postulates:

(1) $x^{\phi}$ is, for every $x$ in $G$, a uniquely determined element in $H$.

(2) $(s x)^{\phi}=s^{\phi} x^{\phi}$ for $s$ in $S$ and $x$ in $G$.

(3) $\phi$ maps $S$ exactly upon the whole group $T$.

(4) $\phi$ induces in $G^{*}=G / S$ a homomorphism $\lambda$ upon the whole group $H^{*}=H / T$.

(5) If $n$ is a positive integer, and if $x$ and $y$ are elements in $G$ and $x^{n}, y^{n}$ elements in $S$, then $\left((x y)^{n}\right)^{\phi}=\left((x y)^{\phi}\right)^{n}=\left(x^{\phi} y^{\phi}\right)^{n}$.

It follows from (2) and (3) that the $S$-T-transformation $\phi$ induces in $S$ a homomorphism $\sigma$ upon $T$, and it follows from (1) to (4) that every element in $H$ is, under $\phi$, the picture of some element in $G$. Using the conditions (1) to (4) we may analyze the condition (5) in the following way. By choosing $y=1,(5)$ specializes to the statement:

† Cf., for example, R. Baer, Mathematische Zeitschrift, vol. 38 (1934), pp. 375-416. 
$\left(5^{\prime}\right)$ If $n$ is a positive integèr, $x$ an element in $G$, and $x^{n}$ an element in $S$, then $\left(x^{n}\right)^{\phi}=\left(x^{\phi}\right)^{n}$.

Since $\phi$ induces a homomorphism of $S$ upon the whole group $T$, it follows that $\left(S^{n}\right)^{\phi}=T^{n}$, consequently $\left(5^{\prime}\right)$ implies the condition:

Condition 4.a. $P\left(S<G ; n, x^{*}\right)^{\sigma}=P\left(T<H ; n, x^{* \lambda}\right)$ for $x^{*}$ in $G_{n}{ }^{*}$.

If (1) to (4) and $\left(5^{\prime}\right)$ hold, and if $S x$ and $S y$ are elements in $G_{n}{ }^{*}$, then

$$
\begin{aligned}
& \left((x y)^{\phi}\right)^{n}=\left((x y)^{n}\right)^{\phi}=\left((y, x)^{n(n-1) 2^{-1}} x^{n} y^{n}\right)^{\phi} \\
& =\left((y, x)^{n(n-1) 2^{-1}}\right)^{\phi}\left(x^{n}\right)^{\phi}\left(y^{n}\right)^{\phi}, \\
& \left(x^{\phi} y^{\phi}\right)^{n}=\left(y^{\phi}, x^{\phi}\right)^{n(n-1) 2^{-1}}\left(x^{\phi}\right)^{n}\left(y^{\phi}\right)^{n} \\
& =\left(y^{\phi}, x^{\phi}\right)^{n(n-1) 2^{-1}}\left(x^{n}\right)^{\phi}\left(y^{n}\right)^{\phi} .
\end{aligned}
$$

This follows from (1.3) and the facts that $\phi$ is a homomorphism of $S$ upon $T$ and of $G^{*}$ upon $H^{*}$, and that $x^{n}, y^{n}$ are in $S$. In view of (1.1) it is fairly obvious that under the assumption of (1) to (4), condition (5) holds if, and only if, condition $\left(5^{\prime}\right)$ and the following condition are satisfied:

Condition 4.b. $\left(\left(S<G ; x^{*}, y^{*}\right)^{2^{m-1}}\right)^{\sigma}=\left(T<H ; x^{* \lambda}, y^{* \lambda}\right)^{2^{m-1}}$ for $x^{*}$ and $y^{*}$ in $G_{2^{m}} *$.

Theorem 4.1. Suppose that $S, G$ and $T, H$ are groups with the property $C(G) \leqq S \leqq Z(G)$ and $C(H) \leqq T \leqq Z(H)$, that $G^{*}=G / S$ is a direct product of cyclic groups, and that conditions 4.a and 4.b are satisfied by the homomorphism $\sigma$ of $S$ upon $T$ and the homomorphism $\lambda$ of $G^{*}$ upon $H^{*}=H / T$. Then there exists an $S$-T-transformation $\gamma$ of $G$ into $H$ which induces $\sigma$ in $S$, and $\lambda$ in $G^{*}$ and satisfies the condition:

(6) If $T^{\prime}$ is the subgroup of $T$, generated by the elements

$$
\left(T<H ; y^{* \lambda}, x^{* \lambda}\right)\left(S<G, x^{*}, y^{*}\right)^{\sigma}
$$

for $x^{*}$ and $y^{*}$ in $G^{*}$, then $\gamma$ induces a homomorphism of $G$ upon $H / T^{\prime}$.

Remark. An obvious consequence of the condition (6) is the following condition:

(6') If $C(G) \leqq U \leqq S$ and $C(H)=U^{\sigma}$, then $\gamma$ induces a homomorphism of $G / U$ upon $H / U^{\sigma}$.

Proof. Let $B$ be an ordered set in $G$ representing a basis of $G / S$. If $b$ is any element in $B$, and if the order of $S b$ is finite, then it follows from Condition 4.a that there exists an element $f(b)$ in $(S b)^{\lambda}$ such that $\left(b^{n(S b)}\right)^{\sigma}=f(b)^{n(S b)}$, where $n(S b)$ is the order of $S b$.

It has been mentioned in $\S 3$ that every element $x$ in $G$ may be represented in one and only one way in the form 


$$
x=s(x) \prod_{b} b^{n(x, b)},
$$

where $s(x)$ is an element in $S$, almost every $n(x, b)=0$, and $0 \leqq n(x, b)<n(S b)$, if $S b$ is an element of finite order $n(S b)$. A function $\gamma$ may be defined for every $x$ in $G$ by the equation

$$
x^{\gamma}=s(x)^{\sigma} \prod_{b \in B} f(b)^{n(x, b)},
$$

where the factors in the product are ordered in the same way as the elements $b$ in $B$.

The element $x^{\gamma}$ is, for every $x$ in $G$, uniquely determined in $H$. If $s$ is an element in $S$, and $x$ in $G$, then $s(s x)=s s(x)$ and $n(s x, b)=n(x, b)$; consequently $(s x)^{\gamma}=s^{\sigma} x^{\gamma}=s^{\gamma} x^{\gamma}$. This implies also that $\gamma$ induces $\sigma$ in $S$. Since $B$ is a basis of $G(\bmod S)$, and since $(S b)^{\gamma}=T f(b)=(S b)^{\lambda}$, it follows that $\gamma$ induces $\lambda$ in $G^{*}$.

Suppose now that $x$ is an element in $G$ and $n$ a positive integer such that $x^{n}$ is an element in $S$. Then $n(x, b)=0$ if $S b$ is of infinite order, and $n(x, b) n$ $=n^{\prime}(x, b) n(S b)$ if $S b$ is of finite order. It follows from (1.3) that

$$
x^{n}=s(x)^{n} a\left(x^{n}\right) c\left(x^{n}\right),
$$

where

$$
a\left(x^{n}\right)=\prod_{b \in B} b^{n(x, b) n}=\prod^{\prime}\left(b^{n(S b)} \cdot\right)^{n^{\prime}(x, b)}
$$

( $\Pi^{\prime}$ indicates a product over those $b$ such that $S b$ is of finite order), and

$$
c\left(x^{n}\right)=\prod_{b<b^{\prime}}\left(S<G ; S b^{\prime}, S b\right)^{n\left(x, b^{\prime}\right) n(x, b) n(n-1) 2^{-1}} .
$$

Computing $a\left(\left(x^{\gamma}\right)^{n}\right)$ and $c\left(\left(x^{\gamma}\right)^{n}\right)$, accordingly, one finds that

$$
a\left(x^{n}\right)^{\gamma}=a\left(x^{n}\right)^{\sigma}=\prod^{\prime} f(b)^{n(S b) n^{\prime}(x, b)}=a\left(\left(x^{\gamma}\right)^{n}\right)
$$

and it follows from Condition 4.b that

$$
c\left(x^{n}\right)^{\gamma}=c\left(x^{n}\right)^{\sigma}=c\left(\left(x^{\gamma}\right)^{n}\right) .
$$

Thus finally

$$
\left(x^{n}\right)^{\gamma}=\left(x^{n}\right)^{\sigma}=\left(s(x)^{\sigma}\right)^{n} a\left(\left(x^{\gamma}\right)^{n}\right) c\left(\left(x^{\gamma}\right)^{n}\right)=\left(x^{\gamma}\right)^{n} .
$$

Hence $\gamma$ satisfies the conditions (1) to (4) and (5'), and, since $\gamma$ satisfies Condition 4.b, this implies that $\gamma$ is an $S$ - $T$-transformation of $G$ into $H$.

If $x$ and $y$ are two elements in $G$, if $a(x, y)$ and $c(x, y)$ are defined as in $\S 3$, and if $a\left(x^{\gamma}, y^{\gamma}\right)$ and $c\left(x^{\gamma}, y^{\gamma}\right)$ are computed accordingly, then

$$
a(x, y)^{\gamma}=a(x, y)^{\sigma}=a\left(x^{\gamma}, y^{\gamma}\right),
$$


and

$$
\begin{aligned}
(x y)^{\gamma} & =\left(s(x) s(y) a(x, y) c(x, y) \prod_{b \in B} b^{n(x y, b)}\right)^{\gamma} \\
& =s(x)^{\sigma} s(y)^{\sigma} a\left(x^{\gamma}, y^{\gamma}\right) c(x, y)^{\sigma} \prod_{b \in B} f(b)^{n(x y, b)} \\
& =c(x, y)^{\sigma} c\left(x^{\gamma}, y^{\gamma}\right)^{-1} x^{\gamma} y^{\gamma} .
\end{aligned}
$$

This shows that $\gamma$ satisfies (6) also.

LEMMA 4.2. If $C(G) \leqq S \leqq Z(G)$, if $C(H) \leqq T \leqq Z(H)$, and if $\phi$ is an $S-T$ transformation of $G$ into $H$ which induces in $S$ the homomorphism $\sigma$ and in $G / S$ the homomorphism $\lambda$, then a necessary and sufficient condition for $\phi$ to be a one-one correspondence is that $\sigma$ and $\lambda$ are isomorphisms.

Proof. The necessity of the conditions is clear. If the conditions are satisfied, and if $x$ and $y$ are two elements in $G$ such that $x^{\phi}=y^{\phi}$, then $(S x)^{\lambda}=(S y)^{\lambda}$, and consequently $S x=S y$; that is, $x=s y$ for some $s$ in $S$. Hence $y^{\phi}=x^{\phi}=(s y)^{\phi}$ $=s^{\sigma} y^{\phi}$ and $x=y$, since $s^{\sigma}=1$ implies $s=1$.

5. Homomorphisms and isomorphisms. We prove the following theorem:

Theorem 5.1. Suppose that $S, G$ and $T, H$ are groups with the property $C(G) \leqq S \leqq Z(G)$ and $C(H) \leqq T \leqq Z(H)$, that $G / S$ is a direct product of cyclic groups, that $\sigma$ is a homomorphism of $S$ upon $T$ and $\lambda$ a homomorphism of $G^{*}=G / S$ upon $H^{*}=H / T$. Then there exists a homomorphism of $G$ into $H$ which induces $\sigma$ in $S$ and $\lambda$ in $G^{*}$ if, and only if,

(a) $\left(S<G ; x^{*}, y^{*}\right)^{\sigma}=\left(T<H ; x^{* \lambda}, y^{* \lambda}\right)$ for $x^{*}, y^{*}$ in $G^{*}$,

(b) $P\left(S<G ; n, x^{*}\right)^{\sigma}=P\left(T<H ; n, x^{* \lambda}\right)$ for $x^{*}$ in $G_{n}{ }^{*}$.

$A$ homomorphism of $G$ into $H$, inducing $\sigma$ in $S$ and $\lambda$ in $G^{*}$, is an isomorphism if, and only if, $\sigma$ and $\lambda$ are isomorphisms.

Proof. The necessity of these conditions is obvious. If, on the other hand, $\sigma$ and $\lambda$ satisfy the conditions (a) and (b), then it follows from Theorem 4.1 that there exists an $S$ - $T$-transformation $\gamma$ of $G$ into $H$ which induces $\sigma$ in $S$ and $\lambda$ in $G^{*}$ and satisfies the condition (6) of Theorem 4.1. But by condition (a) it follows that the subgroup $T^{\prime}$ of $T$, mentioned in (6), consists of the identity only, and $\gamma$ is consequently a homomorphism. The last statement of Theorem 5.1 is an obvious consequence of Lemma 4.2.

6. Uniqueness theorems. We prove the following theorems:

Theorem 6.1. Suppose that $S, G$ and $T, H$ are groups with the property $C(G) \leqq S \leqq Z(G), C(H) \leqq T \leqq Z(H)$, and that $G / S$ is a direct product of cyclic groups. Then there exists an isomorphism of $G$ upon $H$ which maps $S$ upon $T$ if, and only if, there exists an isomorphism $\sigma$ of $S$ upon $T$ and an isomorphism $\lambda$ of $G^{*}=G / S$ upon $H^{*}=H / T$ satisfying conditions (a) and (b) of Theorem 5.1. 
This is an obvious consequence $\dagger$ of Theorem 5.1.

TheOREM 6.2. Suppose that $G / Z(G)$ is a direct product of cyclic groups. Then $G$ and the group $H$ are isomorphic if, and only if, there exists an isomorphism $\sigma$ of $Z(G)$ upon $Z(H)$ and an isomorphism $\lambda$ of $G / Z(G)$ upon $H / Z(H)$ which satisfy the conditions (a) and (b) of Theorem 5.1.

This is a consequence of Theorem 6.1 and the fact that an isomorphism of $G$ upon $H$ maps $Z(G)$ upon $Z(H)$.

Remark 6.3. If one assumes that $G$ and $H$ are groups with abelian central quotient groups, then Theorem 6.2 is transformed into another true theorem, if everywhere the commutator group is substituted for the central.

Remark 6.4. If $G / Z(G)$ is abelian and countable and does not contain elements of infinite order, then $G / Z(G)$ is a direct product of cyclic groups.

Since $G^{*}=G / Z(G)$ is abelian and does not contain elements of infinite order, it is the direct product of its primary components. If $w^{*}$ is an element in $G^{*}$ whose order is a power $p^{i}$ of the prime number $p$, if the equation $y^{*} p^{k}=w^{*}$ has a solution for every positive integer $k$, and if $x^{*}$ is an element of order $p^{i}$ in $G^{*}$, then

$$
\begin{aligned}
\left(Z(G)<G ; w^{*}, x^{*}\right) & =w^{*} \circ x^{*}=v^{* p i} \circ x^{*} \text { for some } v^{*} \epsilon G^{*} \\
& =v^{*} \circ x^{* p^{i}}=1
\end{aligned}
$$

by (1.1). Now it follows from (1.2) that $w^{*}=1$. Since $G^{*}$ is countable, it follows from a theorem of $\mathrm{H}$. Prüfer $\ddagger$ that $G^{*}$ is a direct product of cyclic groups.

In Appendix A a uniqueness theorem will be proved in which $G / Z(G)$ does not contain elements of infinite order and need not be a direct product of cyclic groups.

Example 6.5. This is to show that Theorem 6.2 does not hold if $G / Z(G)$ is a countable abelian group but not a direct product of cyclic groups.

Denote by $A$ a direct product of a countably infinite number of infinite cyclic groups, by $a(1), a(2), \cdots$, a basis of $A$ and by $B$ an abelian group which is generated by elements $b(1), b(2), \cdots$, subject to the relations $b(i+1)^{n}=b(i)$, for $i=1,2, \cdots$, and a fixed integer $n>1 . Z$ is the direct product of $A$ and $B$.

The abelian group $G^{*}$ is generated by elements $g(i, j)^{*}$, for $j=1,2$ and $i=1,2, \cdots$, satisfying the relations $g(i+1, j)^{*_{n}}=g(i, j)^{*}$, for $i=1,2, \cdots$,

$\dagger$ That these conditions are sufficient is an obvious consequence of well known theorems in the theory of extensions of groups; cf., for example, R. Baer, Mathematische Zeitschrift, vol. 38 (1934), p. 410 , formula (3), or p. 391 , Theorem 2.

$\ddagger$ H. Prüfer, Mathematische Zeitschrift, vol. 17 (1923), pp. 48-57. 
$G^{*}$ does not contain elements of finite order not equal to one, and a normal form of the elements in $G^{*}$ is

$$
g(i, 1)^{* h} g(m, 2)^{k},
$$

with $0<h<n$, if $1<i$, and $0<k<n$, if $1<m$.

Essentially the only operation $x^{*} \circ y^{*}$ of $G^{*}$ in $Z$ which satisfies the conditions (1) to (4) of Corollary 2.3 is defined by $g(i, 1)^{* h} g(m, 2)^{* k} \circ g\left(i^{\prime}, 1\right)^{* h^{\prime}} g\left(m^{\prime}, 2\right)^{* k^{\prime}}=b\left(i+m^{\prime}-1\right)^{h k^{\prime}} b\left(i^{\prime}+m-1\right)^{-h^{\prime} k}$.

The group $G$ is generated by adjoining to $Z$ elements $g(i, j)$ which are subject to the relations

$$
Z \leqq Z(G), \quad g(i+1, j)^{n}=g(i, j), \quad(g(i, 1), g(k, 2))=b(i+k-1) .
$$

The group $W$ is generated by adjoining to $Z$ elements $w(i, j)$ which are subject to the relations

$$
Z \leqq Z(W), \quad w(i+1, j)^{n}=w(i, j) a(i), \quad(w(i, 1), \quad w(k, 2))=b(i+k-1) .
$$

It follows from (1.2) that both groups $G$ and $W$ have $Z$ as central and $G^{*}$. as central quotient group, and realize the operation $x^{*} \circ y^{*}$. Since $G^{*}$ does not contain elements other than one of finite order, the conditions of Theorem 6.2 are satisfied with the exception that $G^{*}$ is not a direct product of cyclic groups. But $G$ and $W$ are not isomorphic. For every class, not equal to $Z$, of $G / Z$ contains elements which are $n^{r}$ th powers of elements in $G$ for every positive integer $r$. But if $x$ is an element in the class $Z w(1,1)$ of $W / Z$, then the integers $r$, such that $y^{n r}=x$ has a solution $y$ in $W$, are bounded, as an $n^{r}$ th power of an element in $W$ which is contained in $Z w(1,1)$ has the form

$$
z^{n r} a(r)^{n r-1} a(r-1)^{n-2} \cdots a(1) w(1,1), z \text { in } Z .
$$

It is a consequence of Theorem 2.1 and Theorem 6.2 that the problem to construct all groups whose centrals are a preassigned abelian group $Z$ and whose central quotient groups are isomorphic to a given direct product $G^{*}$ of cyclic groups is equivalent to the problem to define all sets of functions

$$
x^{*} \circ y^{*}, \quad P\left(n, x^{*}\right) \text {. }
$$

which satisfy the conditions (1) to (4) of Corollary 2.3 and the conditions (2), (3) of Theorem 2.1. If

$$
x^{*} \circ y^{*}, \quad P\left(n, x^{*}\right) \text { and } x^{*} \ominus y^{*}, \quad P^{\prime}\left(n, x^{*}\right)
$$

are two sets of functions of $G^{*}$ in $Z$, subject to the mentioned conditions, then they characterize isomorphic groups if, and only if, there exists an automorphism $\zeta$ of $Z$ and an automorphism $\gamma$ of $G^{*}$ such that 
(a) $x^{* \gamma} \circ y^{*} \gamma=\left(x^{*} \ominus y^{*}\right)^{\xi}$ for $x^{*}, y^{*}$ in $G^{*}$, and

(b) $P\left(n, x^{*}\right)=P^{\prime}\left(n, x^{*}\right)^{5}$ for $x^{*}$ in $G_{n}{ }^{*}$.

That it is not sufficient to assume the existence of one pair of automorphisms $\gamma, \zeta$ satisfying (a) and of another pair of automorphisms $\gamma^{\prime}, \zeta^{\prime}$, satisfying (b), may be seen from the following example:

Example 6.6. Suppose that $Z$ is a direct product of a cyclic group of order $p^{3}$, generated by $v$, and a cyclic group of order $p$, generated by $u$, where $p$ is a prime number not 2 , and that $G^{*}$ is a direct product of two cyclic groups of order $p^{2}\left(b^{*}, c^{*}\right.$ a basis of $\left.G^{*}\right)$. Admissible sets of functions of $G^{*}$ in $Z$ may be characterized by the equations

$$
b^{*} \circ c^{*}=v^{p} u, \quad P\left(p^{2}, b^{*}\right)=P\left(p^{2}, c^{*}\right)=Z^{p^{2}} v^{p} u,
$$

and another set by the equations

$$
b^{*} \theta c^{*}=v^{p} u, \quad P\left(p^{2}, b^{*}\right)^{\prime}=P\left(p^{2}, c^{*}\right)^{\prime}=Z^{p^{2} v^{p} u^{-1}} .
$$

Clearly there exists a pair of automorphisms $\gamma, \zeta$ which satisfies (a) and another pair which satisfies (b) but none which satisfies both (a) and (b).

7. Types of subgroups. Suppose that $G$ is a group with abelian central quotient group, and that the subgroups $S$ and $T$ of $G$ are situated between the central and the commutator group of $G$. Assume furthermore that $G / S$ is a direct product of cyclic groups. Then it follows from Theorem 5.1 that there exists an automorphism of $G$ which maps $S$ upon $T$ (that is, that $S$ and $T$ are isotype in $G$ ) if, and only if, there exists an isomorphism $\sigma$ of $S$ upon $T$ and an isomorphism $\lambda$ of $G / S$ upon $G / T$ such that

(a) $\left(S<G ; x^{*}, y^{*}\right)^{\sigma}=\left(T<G ; x^{* \lambda}, y^{* \lambda}\right)$ for $x^{*}, y^{*}$ in $G / S$ and

(b) $P\left(S<G ; n, x^{*}\right)^{\sigma}=P\left(T<G ; n, x^{* \lambda}\right)$ for $x^{*}$ in $(G / S)_{n}$.

If, in particular, $G$ is an abelian group, then condition (a) may be omitted, and this characterization of the types of subgroups applies to any subgroup whose quotient group is a direct product of cyclic groups. It applies therefore to all subgroups, if, for example, $G$ is a group with a finite number of generators or if the orders of the elements in $G$ are bounded.

8. Conformality. Two groups $G$ and $H$ are said to be conformal, if they contain for every positive integer $n$ the same number of elements of order $n$ and also the same number of elements of infinite order. Since (as obvious examples show) infinite abelian groups without elements of infinite order may be conformal without being isomorphic, this definition of conformality is too wide for our purposes, and therefore the following definition may be adopted.

DeFINITION 8.1. The groups $G$ and $H$ with abelian central quotient groups are said to be conformal if there exist groups $S$ and $T$ between the central and the commutator groups of $G$ and $H$, respectively, and an $S$-T-transformation of $G$ 
into $H$ which is a one-one correspondence between $G$ and $H$ and satisfies condition (6) of Theorem 4.1. Such an S-T-transformation may be termed an S-Tconformality of $G$ upon $H$.

Since $S$ - $T$-conformalities induce isomorphisms in $S$ and $G / S$ and satisfy condition $\left(5^{\prime}\right)$ of $\$ 4$, conformal groups are also conformal in the wider sense mentioned above. It is a consequence of condition (6) of Theorem 4.1 that conformalities between abelian groups are isomorphisms.

Conformality is a symmetric and reflexive relation. But in general it is not transitive as follows from Example 8.4.

Suppose now that $C(G) \leqq S \leqq Z(G), C(H) \leqq T \leqq Z(H)$ and that $G / S$ is a direct product of cyclic groups. Then it follows from the results of $\$ 4$, that there exists an $S$ - $T$-conformality of $G$ upon $H$ if, and only if, there exists an isomorphism $\sigma$ of $S$ upon $T$ and an isomorphism $\lambda$ of $G / S$ upon $H / T$ such that

(a) $P\left(S<G ; n, x^{*}\right)^{\sigma}=P\left(T<H ; n, x^{* \lambda}\right)$ for $x^{*}$ in $(G / S)_{n}$ and

(b) $\left(S<G ; x^{*}, y^{*}\right)^{2^{m-1} \sigma}=\left(T<H ; x^{* \lambda}, y^{* \lambda}\right)^{2^{m-1}}$ for $x^{*}, y^{*}$ in $(G / S)_{2^{m}}$.

Exactly those pairs $\sigma, \lambda$ which satisfy the conditions (a) and (b) may be induced by $S-T$-conformalities of $G$ upon $H$.

As condition (b) is not very restrictive, this shows that conformality depends essentially on the $P$-functions.

THEOREM 8.2. If $G$ is a group with abelian.central quotient group, and if $G / C(G)$ is a direct product of cyclic groups, then the following three properties of $G$ are equivalent:

(a) $G$ is conformal to an abelian group.

(b) If $x$ and $y$ are elements of $G$ and if $n$ is a positive integer such that $x^{n}$ and $y^{n}$ are elements of $C(G)$, then $x^{n} y^{n}=(x y)^{n}$.

(c) $\left(C(G)<G ; x^{*}, y^{*}\right)^{2^{m-1}}=1$ for $x^{*}, y^{*}$ in $(G / C(G))_{2^{m}}$.

Proof. If (a) holds, then there exists a group $S$ between $C(G)$ and $Z(G)$, an abelian group $H$ and a subgroup $T$ of $H$, and an $S$ - $T$-conformality $\phi$ of $G$ upon $H$. If $n$ is a positive integer, and if $x$ and $y$ are elements in $G$ such that $x^{n}$ and $y^{n}$ are elements in $C(G)$, then $x^{n}$ and $y^{n}$ are elements in $S$; hence

$$
\begin{aligned}
\left((x y)^{n}\right)^{\phi} & =\left(x^{\phi} y^{\phi}\right)^{n}=\left(x^{\phi}\right)^{n}\left(y^{\phi}\right)^{n} \\
& =\left(x^{n}\right)^{\phi}\left(y^{n}\right)^{\phi}=\left(x^{n} y^{n}\right)^{\phi},
\end{aligned}
$$

since $G^{\phi}=H$ is an abelian group, condition ( $\left.5^{\prime}\right)$ holds, and $\phi$ is a homomorphism on $S$. Now (b) is a consequence of the fact that $\phi$ defines a one-one correspondence between $G$ and $H$.

If (b) holds true, then it follows from (1.3) that for any two elements $x$ and $y$ such that $C(G) x$ and $C(G) y$ are elements of $(G / C(G))_{2^{m}}$ the following equalities hold: 


$$
x^{2^{m}} y^{2^{m}}=(x y)^{2^{m}}=(C(G)<G ; C(G) x, C(G) y)^{2^{m-1}} x^{2^{m}} y^{2^{m}} ;
$$

and $(c)$ is therefore a consequence of $(b)$.

Suppose now that (c) is satisfied. Then it follows from Theorem 2.1 that there exists an abelian group $H$, which contains $C(G)=S$ as a subgroup, and an isomorphism $\gamma$ of $G / C(G)$ upon $H / S$ such that $P\left(S<H ; n, x^{*} \gamma\right)$ $=P\left(C(G)<G ; n, x^{*}\right)$ for $x^{*}$ in $(G / C(G))_{n}$. It follows, from the fact mentioned in the beginning of this section, that there exists a $C(G)-S$-conformality of $G$ upon $H$ which maps every element in $C(G)=S$ upon itself and induces $\gamma$ in $G / C(G)$. Hence (a) is a consequence of (c).

Note that the existence of a basis of $G / C(G)$ has been needed only to prove that (a) is a consequence of (c).

THEOREM 8.3. If $G$ is a group with abelian central quotient group, if $G / C(G)$ is a direct product of cyclic groups, and if $H$ and $H^{\prime}$ are abelian groups which are both conformal to $G$, then $H$ and $H^{\prime}$ are isomorphic.

Proof. From the assumptions there exist subgroups $S$ and $S^{\prime}$ between $C(G)$ and $Z(G)$, an $S$ - $S^{\phi}$-conformality $\phi$ of $G$ upon $H$, and an $S^{\prime}-S^{\prime} \phi^{\prime}$-conformality $\phi^{\prime}$ of $G$ upon $H^{\prime}$. Since $H$ and $H^{\prime}$ are abelian groups, $\phi$ and $\phi^{\prime}$ are isomorphisms in $C(G)$, it follows from condition (6) of Theorem 4.1 that $\phi$ is a

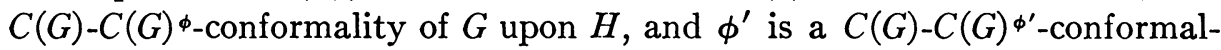
ity of $G$ upon $H^{\prime}$. Consequently there exists an isomorphism $\sigma$ of $C(G)^{\phi}=T$ upon $C(G)^{\phi^{\prime}}=T^{\prime}$ and an isomorphism $\lambda$ of $H / T$ upon $H^{\prime} / T^{\prime}$ such that

$$
P\left(T<H ; n, x^{*}\right)^{\sigma}=P\left(T^{\prime}<H^{\prime} ; n, x^{* \lambda}\right)
$$

for $x^{*}$ in $(H / T)_{n}$. Since $G / C(G), H / T$, and $H^{\prime} / T^{\prime}$ are isomorphic groups and therefore direct products of cyclic groups, and since $H$ and $H^{\prime}$ are abelian groups, it follows from Theorem 5.1 that there exists an isomorphism of $H$ upon $H^{\prime}$ which induces $\sigma$ in $T$ and $\lambda$ in $H / T$.

This proof together with the considerations in $\$ 7$ makes it clear that the investigation of the functions $P\left(n, x^{*}\right)$ is a problem which is equivalent to the problem of characterization of the classes of isotype subgroups of an abelian group.

Example 8.4. This is to show that the Theorem 8.3 is no longer true, when the condition of the existence of a basis of $G / C(G)$ is omitted, and this proves incidentally that the conformality relation is not transitive.

Let $A$ be the direct product of infinite cyclic groups with the basis $a(1)$, $a(2), \cdots$, and let $B$ be the abelian group generated by the elements $b(1)$, $b(2), \cdots$ subject to the relations

$$
b(i+1)^{n}=b(i),
$$


where $1<n$ is a fixed odd integer. $Z$ denotes the direct product of $A$ and $B$.

$A^{*}$ denotes the direct product of infinite cyclic groups with the basis $a^{*}(1), a^{*}(2), \cdots$ and $B^{*}$ the abelian group generated by elements $b^{*}(i, j)$, for $i=1,2, \cdots$ and $j=1,2$, subject to the relations

$$
b^{*}(i+1, j)^{n}=b^{*}(i, j) .
$$

$G^{*}$ denotes the direct product of $A^{*}$ and $B^{*}$.

An operation $x^{*} \circ y^{*}$ of $G^{*}$ in $Z$, satisfying the conditions (1) to (4) of Corollary 2.3 , is characterized by the equations

$$
\begin{aligned}
a^{*}(2 i-1) \circ a^{*}(2 i) & =a(i), \\
a^{*}(i) \circ a^{*}(j) & =1, \text { for } i<j, \quad(i, j) \neq(2 k-1,2 k), \\
b^{*}(i, j) \circ a^{*}(k) & =1, \\
b^{*}(i, 1) \circ b^{*}(k, 2) & =b(i+k-1) .
\end{aligned}
$$

The group $G$ may be the group, generated by adjoining to $Z$ elements $u(1), u(2), \cdots, v(i, j)$, for $i=1,2, \cdots ; j=1,2$, subject to the relations

$$
\begin{aligned}
Z \leqq Z(G), \quad(u(i), u(j)) & =a^{*}(i) \circ a^{*}(j), \quad(u(i), v(k, j))=1, \\
(v(i, 1), v(k, 2)) & =b^{*}(i, 1) \circ b^{*}(k, 2), \\
v(i+1, j)^{n} & =v(i, j) .
\end{aligned}
$$

$G$ is an extension of $Z$ by $G^{*}$ which realizes the operation $x^{*} \circ y^{*}$. Hence $Z=C(G)=Z(G)$.

It is easily seen that $G$ is conformal to the abelian group $H$ which is the direct product of $Z$ and $G^{*}$.

Denote by $U^{\prime}$ the direct product of infinite cyclic groups generated by the elements $u^{\prime}(1), u^{\prime}(2), \cdots$, by $B^{\prime}$ the direct product of the infinite cyclic groups, generated by the elements $b^{\prime}(i, j)$, for $i=1,2, \cdots, j=1,2$, and by $A^{\prime}$ the subgroup of $B^{\prime}$ which is generated by the elements $a^{\prime}(2(i-1)+j)$ $=b^{\prime}(i, j) b^{\prime}(i+1, j)^{-n}$ and is the direct product of the cyclic groups generated by the elements $a^{\prime}(k)$. Let $H^{\prime}$ be the direct product of $U^{\prime}, B^{\prime}$, and $B$, and denote by $Z^{\prime}$ the direct product of $A^{\prime}$ and $B$.

A $Z-Z^{\prime}$-conformality $\phi$ of $G$ upon $H^{\prime}$ is defined by

$$
\begin{aligned}
u^{\prime}(i) & =u(i)^{\phi}, & b^{\prime}(i, j) & =v(i, j)^{\phi}, \\
a^{\prime}(i) & =a(i)^{\phi}, & b(i) & =b(i)^{\phi},
\end{aligned}
$$

since $\phi$ defines an isomorphism of $Z$ upon $Z^{\prime}$ and of $G^{*}$ upon $H^{\prime} / Z^{\prime}$. But $H$ and $H^{\prime}$ are abelian groups which are not isomorphic, $\dagger$ since $H$ is a direct prod-

† Cf. R. Baer, Duke Mathematical Journal, vol. 3 (1937), pp. 69-122, Corollary 2.9. 
uct of cyclic groups by three groups of the type of $B$, whereas $H^{\prime}$ is a direct product of cyclic groups by one group of the type of $B$.

It is a special case of Theorem 8.2 that the group $G$ is conformal to an abelian group, if $C(G) \leqq Z(G)$, and if $G / C(G)$ is a direct product of cyclic groups and does not contain elements of even order. Suppose on the other hand that $C(G) \leqq S \leqq Z(G)$ and that $2^{m}$ with $0<m$ is the l.c.m. of the orders of the elements in $G / S$. If $G$ is $S$ - . - -conformal to an abelian group, and if $w^{*}$ is an element of order $2^{m}$ in $G / S$, then

$$
1=\left(S<G ; w^{*}, x^{*}\right)^{2^{m-1}}=\left(S<G ; w^{* 2^{m-1}}, x^{*}\right)
$$

for every $x^{*}$ in $G / S$; and it follows from (1.1) that the elements in $w^{* 2^{m-1}}$ are elements of $Z(G)$. Since $1 \neq w^{* 2^{m-1}}$, this implies that $S \neq Z(G)$.

It is fairly obvious how to construct groups of order a power of 2 which are conformal to an abelian group. Let, for example, $n$ be an integer, $(1<n), S$ a cyclic group of order $2^{n-1}$ generated by $s$, and $G^{*}$ a direct product of two cyclic groups of order $2^{n},\left(u^{*}, v^{*}\right.$ a basis of $\left.G^{*}\right)$. The group $G$ with $C(G) \leqq S$ $\leqq Z(G)$ which realizes the functions characterized by'

$$
u^{*} \circ v^{*}=s, \quad P\left(2^{n}, u^{*}\right)=P\left(2^{n}, v^{*}\right)=1
$$

is, by Theorem 8.2, conformal to an abelian group, though not itself abelian. If in this construction it had been assumed that $S$ was of order $2^{n}$, then the group $G$ would not be conformal to any abelian group, since $S=C(G)=Z(G)$, and it may be computed that the numbers of the elements of a given order in $G$ are not the same as the corresponding numbers in any abelian group.

9. Groups with isomorphic commutator groups and isomorphic central quotients groups. If $\phi$ is a homomorphism of the group $G$ upon the whole group $H$, then $C(G)^{\phi}=C(H)$ and $Z(G)^{\phi} \leqq Z(H)$. If $S$ is a normal subgroup of $G$, then $S^{\phi}$ is a normal subgroup of $H$, and $\phi$ induces an isomorphism of $G / S$ upon $H / S^{\phi}$, if $S$ is the complete origin of $S^{\phi}$.

Lемма 9.1. If $G$ is a group with abelian central quotient group, and if $\phi$ is a homomorphism of $G$ upon the (whole) group $H$ which induces an isomorphism in $C(G)$, then $Z(G)^{\phi}=Z(H), Z(G)$ is the complete origin of $Z(H)$, and $\phi$ induces an isomorphism in $G / Z(G)$.

Proof. Let $w$ be any element in $G$ such that $w^{\phi}$ is an element in $Z(H)$. If $x$ is any element in $G$, then $(w, x)^{\phi}=\left(w^{\phi}, x^{\phi}\right)=1$, consequently $(w, x)=1$ for every $x$ in $G$; that is, $w$ is an element in $Z(G)$. This proves the lemma.

That the converse does not hold, may be seen from the following example.

Let $p$ be a prime number not 2 , and let $Z$ and $G^{*}$ each be a direct product of three cyclic groups of order $p$. If $z, z^{\prime}$, and $z^{\prime \prime}$ form a basis of $Z$, and $u^{*}, v^{*}$, 
and $w^{*}$ a basis of $G^{*}$, then an operation $x^{*} \circ y^{*}$ of $G^{*}$ in $Z$ which satisfies the conditions (1) to (4) of Corollary 2.3 is characterized by the equations

$$
u^{*} \circ v^{*}=z, \quad v^{*} \circ w^{*}=z^{\prime}, \quad w^{*} \circ u^{*}=z^{\prime \prime} .
$$

The functions $P\left(p, x^{*}\right)=1$, for $x^{*}$ in $G^{*}$, satisfy the conditions of Theorem 2.1 with the operation $x^{*} \circ y^{*}$. It is therefore a consequence of Theorem 2.1, (1.2), and Theorem 6.1 that there exists one, and essentially only one, group $G$ such that $Z=Z(G)$ (or $C(G)$ ) and $G / Z=G^{*}$ which realizes the functions $x^{*} \circ y^{*}$ and $P\left(p, x^{*}\right)$.

Denote by $M$ the subgroup of $G$ generated by $z$, and put $H=G / M$. Then it follows from (1.2) that $Z(H)=C(H)=Z(G) / M$, but this homomorphism of $G$ upon $H$ is not an isomorphism in $C(G)$.

THEOREM 9.2. Suppose that $G$ and $G^{\prime}$ are groups whose central quotient groups are abelian. Then there exists an isomorphism $\psi$ of $C(G)$ upon $C\left(G^{\prime}\right)$ and an isomorphism $\lambda$ of $G / Z(G)$ upon $G^{\prime} / Z\left(G^{\prime}\right)$ with the property that

$$
\left(Z(G)<G ; x^{*}, y^{*}\right)^{\psi}=\left(Z\left(G^{\prime}\right)<G^{\prime} ; x^{* \lambda}, y^{* \lambda}\right)
$$

for $x^{*}, y^{*}$ in $G / Z(G)$ if, and only if, there exists a group $H$ with abelian central quotient group and homomorphisms $\phi$ and $\phi^{\prime}$ of $H$ upon $G$ and $G^{\prime}$, respectively, which induce isomorphisms in $C(H)$.

Proof. Suppose first that there exists a group $H$ with abelian central quotient group and homomorphisms $\phi$ and $\phi^{\prime}$ of $H$ upon $G$ and $G^{\prime}$, respectively, which induce isomorphism in $C(H)$. Then it follows from Lemma 9.1 that $Z(H)^{\phi}=Z(G), Z(H)^{\phi^{\prime}}=Z\left(G^{\prime}\right)$, and that $\phi$ and $\phi^{\prime}$ induce isomorphisms in $H / Z(H)$. Thus $\phi^{-1}$ is an isomorphism in $C(G)$ and in $G / Z(G)$, and $\psi=\phi^{-1} \phi^{\prime}$ is an isomorphism of $C(G)$ upon $C\left(G^{\prime}\right), \lambda=\phi^{-1} \phi^{\prime}$ is an isomorphism of $G / Z(G)$ upon $G^{\prime} / Z\left(G^{\prime}\right)$.

If $x$ and $y$ are two elements in $G$, then there exist some elements $u$ and $v$ in $H$ such that $u^{\phi}=x, v^{\phi}=y$. Consequently

$$
\begin{aligned}
(x, y)^{\psi}=(u, v)^{\phi^{\prime}} & =\left(u^{\phi^{\prime}}, v^{\phi^{\prime}}\right), \\
(Z(G) x, Z(G) y)^{\psi} & =\left((Z(G) x)^{\lambda},(Z(G) y)^{\lambda}\right),
\end{aligned}
$$

and the condition is a sufficient one.

Suppose now that there exists an isomorphism $\psi$ of $C(G)$ upon $C\left(G^{\prime}\right)$ and an isomorphism $\lambda$ of $G / Z(G)$ upon $G^{\prime} / Z\left(G^{\prime}\right)$ such that

(i) $\left(Z(G)<G ; x^{*}, y^{*}\right)^{\psi}=\left(Z\left(G^{\prime}\right)<G^{\prime} ; x^{* \lambda} y^{* \lambda}\right)$ for $x^{*}, y^{*}$ in $G / Z(G)$.

Since $G / C(G)$ is an abelian group, there exist a direct product $G^{*}$ of infinite cyclic groups and a homomorphism $\gamma$ of $G^{*}$ upon $G / C(G)$. There exist by 
Theorem 2.1 and by Theorem 6.1 one, and essentially only one, group $K$, such that $C(K)=C(G) \leqq Z(K)$, and an isomorphism $\kappa$ of $K / C(K)$ upon $G^{*}$ such that

$$
\left(C(K)<K ; x^{*}, y^{*}\right)=\left(C(G)<G ; x^{* \kappa \gamma}, y^{* \kappa \gamma}\right)
$$

for $x^{*}, y^{*}$ in $K / C(K)$. Since $K / C(K)$ is a direct product of infinite cyclic groups, $Z(K) / C(K)$ is also a direct product of infinite cyclic groups, $\dagger$ consequently $Z(K)$ is the direct product of $C(K)$ and a group $D$ which is a direct product of infinite cyclic groups. Since $\kappa \gamma$ is a homomorphism of $K / C(K)$ upon $G / C(G)$, since $K / C(K)$ is a direct product of infinite cyclic groups, it follows from the above equation and Theorem 5.1 that there exists a homomorphism $\rho$ of $K$ upon $G$ which leaves the elements in $C(K)=C(G)$ invariant and induces $\kappa \gamma$ in $K / C(K)$.

Similarly there exists a group $K^{\prime}$, such that $C\left(G^{\prime}\right)=C\left(K^{\prime}\right) \leqq Z\left(K^{\prime}\right)$, $K^{\prime} / C\left(K^{\prime}\right)$ is a direct product of infinite cyclic groups, and $Z\left(K^{\prime}\right)$ is the direct product of $C\left(K^{\prime}\right)$ and a group $D^{\prime}$ which is a direct product of infinite cyclic groups; and there exists a homomorphism $\rho^{\prime}$ of $K^{\prime}$ upon $G^{\prime}$ which leaves all the elements in $C\left(K^{\prime}\right)=C\left(G^{\prime}\right)$ invariant.

Since $G / Z(G)$ is an abelian group, there exists a direct product $H^{*}$ of infinite cyclic groups and a homomorphism $\iota$ of $H^{*}$ upon $G / Z(G)$. Denote by $S$ the direct product of $D, D^{\prime}$, and $C(G)$, and denote by $H$ the essentially uniquely determined group such that $C(H) \leqq S \leqq Z(H)$ and such that there exists an isomorphism $\tau$ of $H / S$ upon $H^{*}$ which satisfies the equation

$$
\left(S<H ; x^{*}, y^{*}\right)=\left(Z(G)<G ; x^{* \tau \iota}, y^{* \tau \iota}\right)
$$

for $x^{*}, y^{*}$ in $H / S$.

Since $H / S$ is a direct product of infinite cyclic groups, $H / C(H)$ is the direct product of $S / C(H)$ and a direct product $D^{*}$ of infinite cyclic groups.

By Lemma 9.1, $\rho$ induces an isomorphism of $K / Z(K)$ upon $G / Z(G)$; consequently the isomorphism $\rho^{-1}$ is well defined on $G / Z(G)$. Since $D^{*}$ is a direct product of infinite cyclic groups and since $D^{*}$ represents exactly $H / S$, there exists a homomorphism $\sigma$ of $H / C(H)$ upon $K / C(K)$ with the following properties: $x^{*_{\sigma}}=1$, if $x^{*}=C(H) d^{\prime}$ and $d^{\prime}$ in $D^{\prime} ; x^{*_{\sigma}}=C(K) d$, if $x^{*}=C(H) d$ and $d$ is an element of $D$; and $Z(K)^{*_{\sigma}}=\left(S x^{*}\right)^{\text {rip }}{ }^{-1}$, if $x^{*}$ is an element of $D^{*}$.

Since $\rho$ induces $\kappa \gamma$ in $K / C(K)$, it follows that

$$
\left(S<H ; x^{*}, y^{*}\right)=\left(Z(K)<K ; x^{* \tau \iota \rho^{-1}}, y^{* \tau \iota \rho^{-1}}\right)
$$

for $x^{*}, y^{*}$ in $H / S$. The homomorphism $\sigma$ therefore satisfies

$\dagger$ R. Baer, Duke Mathematical Journal, vol. 3 (1937), pp. 69-122, especially the remark to Corollary 8.9. 


$$
\left(C(K)<K ; x^{* \sigma}, y^{* \sigma}\right)=\left(C(H)<H ; x^{*}, y^{*}\right)
$$

for $x^{*}, y^{*}$ in $H / C(H)$.

By Lemma 9.1, $\rho^{\prime}$ induces an isomorphism of $K^{\prime} / Z\left(K^{\prime}\right)$ upon $G^{\prime} / Z\left(G^{\prime}\right)$; consequently the isomorphism $\rho^{\prime-1}$ is well defined on $G^{\prime} / Z\left(G^{\prime}\right)$. There exists, therefore, a homomorphism $\sigma^{\prime}$ of $H / C(H)$ upon $K^{\prime} / C\left(K^{\prime}\right)$ with the following properties: $x^{*_{\sigma^{\prime}}}=C\left(K^{\prime}\right) d^{\prime}$, if $x^{*}=C(H) d^{\prime}$ and $d^{\prime}$ is an element in $D^{\prime} ; x^{*_{\sigma^{\prime}}}=1$, if $x^{*}=C(H) d$ and $d$ is an element in $D$; and $Z\left(K^{\prime}\right) x^{*_{\sigma^{\prime}}}=\left(S x^{*}\right)^{r \imath \lambda \rho^{\prime-1}}$, if $x^{*}$ is an element of $D^{*}$.

Since $\rho^{\prime}$ is a homomorphism of $K^{\prime}$ upon $G^{\prime}$ which leaves all the commutators invariant, it follows from condition (i) that

$$
\begin{aligned}
\left(C\left(K^{\prime}\right)<K^{\prime} ; x^{* \sigma^{\prime}}, y^{* \sigma^{\prime}}\right) & =\left(Z\left(K^{\prime}\right)<K^{\prime} ; Z\left(K^{\prime}\right) x^{* \sigma^{\prime}}, Z\left(K^{\prime}\right) y^{* \sigma^{\prime}}\right) \\
& =\left(Z\left(K^{\prime}\right)<K^{\prime} ;\left(S x^{*}\right)^{\tau \imath \lambda \rho^{\prime-1}},\left(S y^{*}\right)^{\tau \imath \lambda \rho^{\prime-1}}\right) \\
& =\left(Z\left(G^{\prime}\right)<G^{\prime} ;\left(S x^{*}\right)^{\tau \iota \lambda},\left(S y^{*}\right)^{\tau \iota \lambda}\right) \\
& =\left(Z(G)<G ;\left(S x^{*}\right)^{\tau \iota},\left(S y^{*}\right)^{\tau \iota}\right)^{\psi} \\
& =\left(S<H ; S x^{*}, S y^{*}\right)^{\psi}=\left(C(H)<H ; x^{*}, y^{*}\right)^{\psi}
\end{aligned}
$$

for every $x^{*}, y^{*}$ in $H / C(H)$.

Since $H / C(H)$ is a direct product of infinitè cyclic groups, it follows from Theorem 5.1 that there exists a homomorphism $\beta$ of $H$ upon $K$ which induces $\sigma$ in $H / C(H)$ and leaves the elements in $C(H)=C(K)$ invariant, and that there exists a homomorphism $\beta^{\prime}$ of $H$ upon $K^{\prime}$ which induces $\sigma^{\prime}$ in $H / C(H)$ and $\psi$ in $C(H)=C(G)$. $\beta \rho$ and $\beta^{\prime} \rho^{\prime}$ are homomorphisms of $H$ upon $G$ and $G^{\prime}$, respectively. $\beta \rho$ leaves the elements in $C(\dot{H})=C(K)=C(G)$ invariant, and $\beta^{\prime} \rho^{\prime}$ induces in $C(H)$ the isomorphism $\psi$. This completes the proof.

In fact slightly more has been proved, namely, that the group $H$, which meets the requirements of the Theorem 9.2, may always be chosen in such a way that $H / C(H)$ is a direct product of infinite cyclic groups.

The proof of this theorem shows the advantage of treating finite and infinite groups at the same time. For even if the groups $G$ and $G^{\prime}$ of the theorem are finite, it may prove difficult to construct a group $H$ which meets all the requirements without recourse to infinite groups.

10. Automorphisms. If $G$ is a group with abelian central quotient group and $S$ a subgroup of $G$ such that $C(G) \leqq S \leqq Z(G)$, then denote by $\Theta(S<G)$ the group of all those (proper) automorphisms of $G$ which map $S$ upon itself. If $S$ is, for example, the central or the commutator group of $G$, then $\Theta(S<G)$ is the group of all automorphisms of $G$.

Those automorphisms of $G$ which leave invariant every element in $S$ and every element in $G / S$ form a normal subgroup $\Omega(S<G)$ of $\Theta(S<G)$. If $\phi$ is 
any automorphism in $\Omega(S<G), x$ an element in $G$ and $s$ an element in $S$, then

$$
x^{\phi} x^{-1}=(s x)^{\phi}(s x)^{-1}=(S x)^{\phi}(S x)^{-1}=(\phi, S x)
$$

is an element in $S$; and if $x$ and $y$ are two elements in $G$, then

$$
\begin{aligned}
(\phi, S x y) & =(x y)^{\phi}(x y)^{-1}=x^{\phi} y^{\phi} y^{-1} x^{-1} \\
& =x^{\phi}(\phi, S y) x^{-1}=(\phi, S x)(\phi, S y),
\end{aligned}
$$

and conversely every homomorphism of $G / S$ into $S$ may be realized this way. Since finally

$$
\begin{aligned}
(\phi \gamma, S x) & =x^{\phi \gamma} x^{-1}=x^{\phi \gamma}\left(x^{-1}\right)^{\gamma} x^{\gamma} x^{-1} \\
& =(\phi, S x)^{\gamma}(\gamma, S x)=(\phi, S x)(\gamma, S x),
\end{aligned}
$$

as $\gamma$ belongs to $\Omega(S<G)$ and $(\phi, S x)$ to $S$, it follows that $\Omega(S<G)$ is essentially equal to the group of all homomorphisms of $G / S$ into $S$.

Two automorphisms in $\Theta(S<G)$ belong to the same class of $\Lambda(S<G)$ $=\Theta(S<G) / \Omega(S<G)$ if, and only if, they indice the same automorphisms in $S$ and in $G / S$. Thus $\Lambda(S<G)$ is essentially equal to a subgroup of the group $\Pi(S, G / S)$ of all the pairs $(\sigma, \lambda)$ of automorphisms $\sigma$ of $S$ and $\lambda$ of $G / S$. If the pair $(\sigma, \lambda)$ in $\Pi(S, G / S)$ is realized by an automorphism in $\Theta(S<G)$, then it follows that

(a) $\left(S<G ; x^{*}, y^{*}\right)^{\sigma}=\left(S<G ; x^{* \lambda}, y^{* \lambda}\right)$ for $x^{*}, y^{*}$, in $G / S$, and

(b) $P\left(S<G ; n, x^{*}\right)^{\sigma}=P\left(S<G ; n, x^{* \lambda}\right)$ for $x^{*}$ in $(G / S)_{n}$;

and these conditions are sufficient for the realizability, provided $G / S$ is a direct product of cyclic groups, as follows from Theorem 5.1.

Suppose now that $\phi$ is an automorphism in $\Omega(S<G)$ and that the automorphism $\gamma$ of $G$ induces in $S$ the automorphism $\sigma$ and in $G / S$ the automorphism $\lambda$. Then

$$
\begin{aligned}
\left(\gamma^{-1} \phi \gamma, S x\right) & =x^{\gamma^{-1} \phi \gamma} x^{-1}=\left(x^{\gamma^{-1} \phi}\left(x^{\gamma^{-1}}\right)^{-1}\right)^{\gamma} \\
& =\left(\phi, S x^{\gamma^{-1}}\right)^{\gamma}=\left(\phi,(S x)^{\lambda^{-1}}\right)^{\sigma}
\end{aligned}
$$

consequently it follows that the class of $\Lambda(S<G)$ which is characterized by the pair $(\sigma, \lambda)$ in $\Pi(S, G / S)$ induces, in the abelian group $\Omega(S<G)$, the automorphism defined by

$$
\left(\phi^{(\sigma, \lambda)}, x^{*}\right)=\left(\phi, x^{* \lambda^{-1}}\right)^{\sigma}
$$

for $x^{*}$ in $G / S$.

In order to characterize the extension $\Theta(S<G)$ of $\Omega(S<G)$ by $\Lambda(S<G)$ which realizes the above mentioned automorphisms in $\Omega(S<G)$, it is necessary to compute the so-called factor sets. A method for their calculation may be indicated for groups $G, S$ which satisfy the conditions: 
(i) $C(G) \leqq S \leqq Z(G)$.

(ii) $G / S=G^{*}$ is a direct product of cyclic groups.

(iii) If $x$ and $y$ are elements of $G$ and $n$ is a positive integer such that $x^{n}$ and $y^{n}$ are elements of $S$, then $x^{n} y^{n}=(x y)^{n}$.

First we define in $S$ for every positive integer $n$ a function $s^{n^{-1}}$ as follows: $1=1^{n^{-1}}$; if the equation $x^{n}=s$ has a solution in $S$, then $s^{n^{-1}}$ is one of these solutions $x$; if $s$ is not contained in $S^{n}$, then $s^{n^{-1}}$ is not defined.

Second an ordered set $B$ is chosen in $G$ which represents exactly a basis $B^{*}$ of $G / S$, and the functions

$$
s(x), \quad n(x, b), \quad a(x, y)=a(S x, S y), \quad c(x, y)=c(S x, S y),
$$

for $x, y$ in $G$ and $b$ in $B$, introduced in $\$ 3$, are defined with reference to the ordered set $B$.

In defining another multiplication of the elements of $G$ by the equation

$$
x^{*} y=c(x, y)^{-1} x y,
$$

for $x, y$ in $G, G$ is transformed into an abelian group $H$, and $S$ is a subgroup of $H$, since $c(x, y)=1$ for $x, y$ in $S$. Thus the identical mapping of the elements in $G$ is an $S$-S-conformality of $G$ upon $H$. Furthermore $G / S=H / S$ and $P\left(S<G ; n, x^{*}\right)=P\left(S<H ; n, x^{*}\right)$ for $x^{*}$ in $G_{n}{ }^{*}$. Finally $\Omega(S<G)=\Omega(S<H)$ and $\Lambda(S<G) \leqq \Lambda(S<H)$, as follows from the conditions (a) and (b) mentioned above.

Suppose now that $(\sigma, \lambda)$ is a pair in $\Pi(S, G / S)$ which may be realized by some automorphism in $\Theta(S<G)$. Then

$$
b^{* \lambda}=\prod_{d^{*} \in B^{*}} d^{* n\left(b_{j^{*}}^{*}, d^{*}, \lambda\right)},
$$

where almost every $n\left(b^{*}, d^{*}, \lambda\right)=0$ and $0 \leqq n\left(b^{*}, d^{*}, \lambda\right)<n\left(d^{*}\right)$, if $d^{*}$ is of finite order $n\left(d^{*}\right)$. If $b^{*}$ is an element of finite order $n=n\left(b^{*}\right)$, and if $d$ in $B$ always represents the element $d^{*}$ in $B^{*}$, then it follows from the realizability of $(\sigma, \lambda)$ that the elements

$$
\left(b^{n}\right)^{\sigma} \prod_{d \in B} d^{-n n\left(b^{*}, d^{*}, \lambda\right)}=s(b, \sigma, \lambda)
$$

are $n$th powers of elements in $S$. Consequently, an automorphism $\gamma=\gamma(\sigma, \lambda)$ in $\Theta(S<G)$ is defined by the equations

$$
\begin{aligned}
& x^{\gamma}=x^{\sigma} \text { for } x \text { in } S, \\
& b^{\gamma}=t(b, \sigma, \lambda) \prod_{d \in B} d^{n\left(b^{*}, d^{*}, \lambda\right)},
\end{aligned}
$$


where $t(b, \sigma ; \lambda)=1$, if $b^{*}$ is of infinite order, and $t(b, \sigma, \lambda)=s(b, \sigma, \lambda)^{n\left(b^{*}\right)^{-1}}$, if $b^{*}$ is of the finite order $n\left(b^{*}\right)$.

It is easily seen that there exists an automorphism in $\Theta(S<H)$ which has the same values as $\gamma$ on $S$ and on $B$. But these automorphisms do not constitute the same mapping of the elements which form both groups $G$ and $H$.

Thus a representative of every class of $\Lambda(S<G)$ has been chosen, and the factor sets are expressions

$$
\gamma(\sigma, \lambda) \gamma\left(\sigma^{\prime}, \lambda^{\prime}\right) \gamma\left(\sigma \sigma^{\prime}, \lambda \lambda^{\prime}\right)^{-1}=a\left(\sigma, \lambda ; \sigma^{\prime}, \lambda^{\prime}\right) c\left(\sigma, \lambda ; \sigma^{\prime}, \lambda^{\prime}\right)
$$

where $a(\cdots)$ is the factor set, calculated for the group $H$, and $c(\cdots)$ is an expression in the $\left(S<G ; b^{*}, d^{*}\right)$.

If all the elements of $G / Z(G)$ are of finite odd order, then the investigations of Appendix B will produce better results. But the methods of Appendix $B$ break down in some of those cases in which the method of this section may be applied.

Appendix A. A uniqueness theorem for $p$-adic groups. It is the object of this appendix to prove a uniqueness theorem which is not contained in Theorem 6.2. Because of Example 6.5 only groups without elements of infinite order will be considered, and by Remark 6.4 it will be necessary to consider groups whose central quotient group is infinite but not countable.

Groups whose central quotient group is abelian are direct products of $p$-groups (that is, groups which contain only elements of order a power of the prime number $p$ ) if, and only if, they do not contain elements of infinite order. $\dagger$ Consequently only $p$-groups will be considered in this appendix.

The $p$-group $G$ will be called $p$-adic if the cross cut of its subgroups $G^{p^{i}}$, for $i=1,2, \cdots$, consists of the group unit element only. The $p$-group $G$ is said to be dense in the p-group $H$, if $G \leqq H$ and every class of $H / H^{p}$ contains elements of $G$. Finally $G$ is termed a closed p-adic group if $G$ is $p$-adic and if $G$ is the only $p$-adic group in which $G$ is dense.

Lemma A.1. (a) Every p-adic group is dense in one and essentially only one closed p-adic group.

(b) If the p-adic group $G$ is dense in the closed p-adic group $\bar{G}$, and if the $p$-adic group $H$ is dense in the closed p-adic group $\bar{H}$, then every isomorphism $\gamma$ of $G$ upon the whole group $H$ is induced by one and only one isomorphism of $\bar{G}$ upon $\bar{H}$.

† Cf., for example, R. Baer, Compositio Mathematica, vol. 1 (1934), pp. 254-283, especially the lemma on p. 261. 
Similar statements have been proved before by similar methods, $\dagger$ and it will therefore be sufficient to give the following indication of a proof of this lemma. If $G$ is a $p$-adic group, then consider the set $S(G)$ of all the sequences $S_{i}$ with the following properties:

(a) $S_{i}$ is a class of $G / G^{p^{i}}$.

(b) $S_{i+1} \leqq S_{i}$.

(c) The orders of the elements $S_{i}$ of the groups $G / G^{p^{i}}$ are bounded. (The upper bounds for the orders may be different for different sequences.)

If the multiplication in $S(G)$ is defined in the obvious way, $S(G)$ becomes a closed $p$-adic group. In mapping the element $x$ of $G$ upon the sequence $G^{p^{i}} x$ an isomorphism of $G$ upon a dense subgroup of $S(G)$ is defined. Now it is fairly obvious how to work out a proof of the lemma.

If $G$ is a $p$-adic group, then the essentially uniquely determined closed $p$-adic group $\bar{G}$, in which $G$ is dense, may be called the $p$-adic closure of $G$.

The following example shows the existence of closed $p$-adic groups whose central quotient group is abelian but not a direct product of cyclic groups.

Denote by $Z_{i}$ and $Z_{i}{ }^{*}$ cyclic groups of order $p^{i}$, generated by $z_{i}$ and $z_{i}^{*}$, respectively; and let $Z$ be the direct product of the groups $Z_{i}$, for $i=1,2, \cdots$, and $Z^{*}$ the direct product of the groups $Z_{i}^{*}$, for $i=1,2, \cdots$. An operation $x^{*} \circ y^{*}$ and functions $P\left(p^{i}, x^{*}\right)$ of $Z^{*}$ in $Z$, which satisfy the conditions (1) to (4) of Corollary 2.3 and the conditions (1) to (3) of Theorem 2.1, may be defined by the following equations:

$$
z_{i}^{*} \circ z_{i+1}^{*}=z_{i}, \quad z_{i}^{*} \circ z_{i+h}^{*}=1 \quad(\text { for } 1<h) ; \quad P\left(p^{i}, z_{i}^{*}\right)=1 .
$$

Thus there exists one, and essentially only one, group $G$ which satisfies $Z=C(G)=Z(G), Z^{*}=G / Z$, and which realizes the functions $x^{*} \circ y^{*}, P\left(p^{i}, x^{*}\right)$. $G$ is clearly a $p$-adic group.

If $\bar{G}$ is the $p$-adic closure of $G$, then $Z(\bar{G})$ is the $p$-adic closure of $Z(G)$, and $\bar{G} / Z(\bar{G})$ is the $p$-adic closure of $G / Z(G)$. It is well known that these abelian groups are not direct products of cyclic groups.

THEOREM A.2. Suppose that $G$ is a closed p-adic group with abelian central quotient group. Then $G$ and $H$ are isomorphic groups if, and only if,

(1) $H$ is a closed p-adic group;

(2) there exists an isomorphism $\zeta$ of $Z(G)$ upon $Z(H)$ and an isomorphism $\lambda$ of $G / Z(G)$ upon $H / Z(H)$ which satisfy the conditions:

(a) $\left(Z(G)<G ; x^{*}, y^{*}\right)^{5}=\left(Z(H)<H ; x^{* \lambda}, y^{* \lambda}\right)$ for $x^{*}, y^{*}$ in $G / Z(G)$;

(b) $P\left(Z(G)<G ; p^{i}, x^{*}\right)^{5}=P\left(Z(H)<H ; p^{i}, x^{* \lambda}\right)$ for $x^{*}$ in $(G / Z(G))_{p^{i}}$.

$\dagger$ †. Baer, Journal für die reine und angewandte Mathematik, vol. 160 (1929), pp. 208-226. H. Freudenthal, Compositio Mathematica, vol. 4 (1937), pp. 145-234. 
If condition (1) is satisfied by $H$, then exactly those pairs of isomorphisms $\zeta, \lambda$ which satisfy (a) and (b) may be induced by isomorphisms of $G$ upon $H$.

Proof. The necessity of the conditions appearing in either of the statements of the theorem is fairly obvious. Suppose now that condition (1) is satisfied by $H$, and that $\zeta, \lambda$ is a pair of isomorphisms satisfying the conditions (a) and (b). It shall be proved that there exists an isomorphism of $G$ upon $H$ which induces $\zeta$ in $Z(G)$ and $\lambda$ in $G / Z(G)$.

Since $G^{*}=G / Z(G)$ is an abelian $p$-group, there exists a direct product $D^{*}$ of cyclic groups which is dense in $G^{*} . \dagger$ Then $D^{* \lambda}$ is dense in the abelian $p$-group $G^{* \lambda}=H / Z(H)$. If $D$ is the group such that $Z(G) \leqq D \leqq G, D / Z(G)$ $=D^{*}$, and if $D^{\prime}$ is the group such that $Z(H) \leqq D^{\prime} \leqq H$, and $D^{\prime} / Z(H)=D^{* \lambda}$, then $D$ is dense in $G$ and $D^{\prime}$ is dense in $H$. It is now a consequence of Theorem 5.1 and the conditions (a), (b) that there exists an isomorphism $\delta$ of $D$ upon $D^{\prime}$ which induces $\zeta$ in $Z(G)$ and $\lambda$ in $D^{*}$; and it follows from Lemma A.1, (b) that there exists a uniquely determined isomorphism $\gamma$ of $G$ upon the closed $p$-adic group $H$ which induces $\delta$ in $D$. Clearly $\gamma$ induces $\zeta$ in $Z(G)$.

Applying the argument used in Remark 6.4 we can show that $G / Z(G)$ is a $p$-adic group. Consequently there exists at most one isomorphism of $G / Z(G)$ upon $H / Z(H)$ which induces a given isomorphism in the group $D^{*}$, dense in $G^{*}$. Since $\gamma$ and $\lambda$ are equal isomorphisms on $D^{*}$, this implies, therefore, that $\gamma$ induces $\lambda$ in the whole group $G / Z(G)$.

It may be noted that by the method applied in the proof even the following slightly more general statements may be proved:

Corollary A.3. Suppose that $G$ and $H$ are closed p-adic groups and that $C(G) \leqq S \leqq Z(G), C(H) \leqq T \leqq Z(H)$. Then there exists an isomorphism of $G$ upon $H$ which maps $S$ upon $T$ if, and only if, there exists an isomorphism $\sigma$ of $S$ upon $T$ and an isomorphism $\lambda$ of $G / S$ upon $H / T$ which satisfy the conditions (a) and (b) of Theorem 5.1. If furthermore $G / S$ is a p-adic group, then exactly the pairs $\sigma, \lambda$, satisfying (a) and (b) of Theorem 5.1 may be realized by isomorphisms of $G$ upon $H$ which map $S$ upon $T$.

Appendix B. Conformalities which preserve the automorphisms. We prove the following theorem:

THEOREM B.1. If $G$ is a group with abelian central quotient group, if the subgroup $S$ of $G$ does not contain elements of order 2 , and if $C(G) \leqq S^{2} \leqq S \leqq Z(G)$, then there exists an abelian group $H$ and a one-one correspondence $\phi$ which maps $G$ upon the whole group $H$ and satisfies the conditions:

(1) $(x y)^{\phi}=x^{\phi} y^{\phi}$ for $x$ in $Z(G)$ and $y$ in $G$.

† R. Baer, American Journal of Mathematics, vol. 59 (1937), pp. 99-117, §1. 
(2) $\left(x^{n}\right)^{\phi}=\left(x^{\phi}\right)^{n}$ for $x$ in $G$ and any integer $n$.

(3) $\phi$ induces a homomorphism of $G$ upon $H / S^{\phi}$.

(4) If $\gamma$ is a (proper or improper) automorphism of $G$ such that $S^{\gamma} \leqq S$, then $\phi^{-1} \gamma \phi$ is an automorphism of $H$.

Note that $\phi$ induces isomorphisms in $Z(G)$ and in $G / S$.

Proof. $†$ Since $C(G) \leqq S^{2}$, and since $S$ does not contain elements of order 2, there exists to every pair $x, y$ of elements in $G$ a uniquely determined element $f(x, y)$ in $S$ such that $f(x, y)^{2}=(x, y)$. Since $f(x, y)$ is the only solution of this equation in $S$, and since $S$ is an abelian group, it follows that

$$
f(x, x)=f(x, y) f(y, x)=1, \quad f(x, y z)=f(x, y) f(x, z) ;
$$

and

$$
f(x, y)=1
$$

if, and only if, $(x, y)=1$.

Denote now by $\phi$ a one-one correspondence which maps $G$ upon a set $H$ of elements. In $H$ a multiplication may be defined by the equation

$$
x^{\phi} y^{\phi}=(f(y, x) x y)^{\phi}
$$

for $x$ and $y$ in $G$. The product of any two elements in $H$ is uniquely determined by this definition. Since $f(x, y)=1$ for $x$ in $Z(G)$, this correspondence $\phi$ between the group $G$ and the multiplicative manifold $H$ satisfies condition (1); and it satisfies (2), since $f\left(x^{i}, x^{j}\right)=1$ for $x$ in $G$ and any integers $i$ and $j$. This implies in particular that the picture of the group unit in $G$ is the uniquely determined unit in $H$, and that the inverse of any element in $H$ is uniquely determined. That the multiplication in $H$ is commutative follows from

$$
x^{\phi} y^{\phi}=(f(y, x) x y)^{\phi}=(f(y, x)(x, y) y x)^{\phi}=(f(x, y) y x)^{\phi}=y^{\phi} x^{\phi} ;
$$

and that the multiplication in $H$ is associative, follows from

and

$$
\begin{aligned}
\left(x^{\phi} y^{\phi}\right) z^{\phi} & =(f(y, x) x y)^{\phi} z^{\phi}=(f(z, f(y, x) x y) f(y, x) x y z)^{\phi} \\
& =f(y, x)^{\phi} f(z, x)^{\phi} f(z, y)^{\phi}(x y z)^{\phi}
\end{aligned}
$$

$$
\begin{aligned}
x^{\phi}\left(y^{\phi} z^{\phi}\right) & =x^{\phi}(f(z, y) y z)^{\phi}=(f(f(z, y) y z, x) x f(y, z) y z)^{\phi} \\
& =f(y, z)^{\phi} f(z, x)^{\phi} f(y, x)^{\phi}(x y z)^{\phi} .
\end{aligned}
$$

Consequently $H$ is an abelian group and $\phi$ satisfies (3), since all the elements $f(x, y)$ are contained in $S$.

$\dagger$ The idea for this proof has been suggested by an argument used by C. Hopkins for the proof of a similar theorem, cf. C. Hopkins, these Transactions, vol. 37 (1935), pp. 169-170. 
Suppose finally that $\gamma$ is an automorphism of $G$ such that $S^{\gamma} \leqq S$. Then

$$
\left(f(x, y)^{\gamma}\right)^{2}=\left(f(x, y)^{2}\right)^{\gamma}=(x, y)^{\gamma}=\left(x^{\gamma}, y^{\gamma}\right)=f\left(x^{\gamma}, y^{\gamma}\right)^{2},
$$

consequently

$$
f(x, y)^{\gamma}=f\left(x^{\gamma}, y^{\gamma}\right)
$$

since $f(x, y)^{\gamma}$ is an element of $S$. Hence

$$
\begin{aligned}
\left(x^{\phi} y^{\phi}\right)^{\phi^{-1} \gamma \phi} & =(f(y, x) x y)^{\gamma \phi}=\left(f(y, x)^{\gamma} x^{\gamma} y^{\gamma}\right)^{\phi} \\
& =\left(f\left(y^{\gamma}, x^{\gamma}\right) x^{\gamma} y^{\gamma}\right)^{\phi}=x^{\gamma \phi} y^{\gamma \phi} \\
& =\left(x^{\phi}\right)^{\phi^{-1} \gamma \phi}\left(y^{\phi}\right)^{\phi^{-1} \gamma \phi}
\end{aligned}
$$

and this completes the proof of the Theorem.

Example B.2. This example is to show that the Theorem B.1 would not hold without the assumptions concerning the existence of a subgroup $S$ without elements of order 2 such that $C(G) \leqq S^{2} \leqq S$.

Denote by $Z$ an infinite cyclic group generated by an element $z$, and by $G^{*}$ a direct product of two infinite cyclic groups. If $u^{*}, v^{*}$ is any basis of $G^{*}$, then an operation $x^{*} \circ y^{*}$ of $G^{*}$ in $Z$ which satisfies the conditions (1) to (4) of Corollary 2.3 is characterized by $u^{*} \circ v^{*}=z$. Let $G$ be the (essentially uniquely determined) group such that $Z=C(G)=Z(G), G^{*}=G / Z$ which realizes the operation $x^{*} \circ y^{*}$.

This group is conformal to a direct product of three infinite cyclic groups.

Suppose that $\phi$ is a one-one correspondence which maps $G$ upon some group $H$ and which satisfies the conditions:

(i) $(x y)^{\phi}=x^{\phi} y^{\phi}$ for $x$ in $Z(G)$ and $y$ in $G$.

(ii) $\left(x^{n}\right)^{\phi}=\left(x^{\phi}\right)^{n}$ for $x$ in $G$ and any integer $n$.

(iii) $\phi$ induces a homomorphism of $G$ upon $H / Z(G)^{\phi}$.

(iv) If $\gamma$ is a proper automorphism of $G$, then $\phi^{-1} \gamma \phi$ is an automorphism of $H$.

Note that (i) and (ii) are identical with the conditions (1) and (2) of the Theorem B.1, whereas (iii) and (iv) are even weaker conditions than the corresponding conditions (3) and (4) of the Theorem B.1.

Since $\phi$ is a one-one correspondence, an element $f(x, y)$ is uniquely determined by the equation

$$
x^{\phi} y^{\phi}=(f(y, x) x y)^{\phi}
$$

for $x$ and $y$ in $G$, and it is a consequence of (iii) that $f(y, x)$ is an element of $Z(G)$. It is a consequence of (i) that $f(x, y)=f(Z(G) x, Z(G) y)$ is independent of the choice of $x$ and $y$ in their respective classes in $G / Z(G)$; and it is a consequence of (ii) that $f\left(x^{i}, x^{j}\right)=1$ for $x$ in $G$ and integral $i$ and $j$. 
Let now $u$ and $v$ be some representatives of the classes $u^{*}$ and $v^{*}$ of $G / Z$, respectively. Then an automorphism $\gamma$ of $G$ is defined by

$$
u^{\gamma}=u v, \quad v^{\gamma}=v^{-1}, \quad z^{\gamma}=z^{-1},
$$

since $\left(u^{\gamma}, v^{\gamma}\right)=\left(u v, v^{-1}\right)=(v, u)=z^{-1}=z^{\gamma}=(u, v)^{\gamma}$. It is therefore a consequence of condition (iv) that

$$
\begin{aligned}
\left(f(v, u)^{\gamma} u^{\gamma} v^{\gamma}\right)^{\phi} & =(f(v, u) u v)^{\gamma \phi}=(f(v, u) u v)^{\phi \phi^{-1} \gamma \phi} \\
& =\left(u^{\phi} v^{\phi}\right)^{-1} \gamma \phi=u^{\phi \phi^{-1} \gamma \phi} v^{\phi \phi^{-1}} \gamma \phi=u^{\gamma \phi} v^{\gamma \phi} \\
& =\left(u^{\gamma}\right)^{\phi}\left(v^{\gamma}\right)^{\phi}=\left(f\left(v^{\gamma}, u^{\gamma}\right) u^{\gamma} v^{\gamma}\right)^{\phi} ;
\end{aligned}
$$

and this implies, since $f(u, v)$ is an element of $Z$, that

$$
f(v, u)^{-1}=f(v, u)^{\gamma}=f\left(v^{\gamma}, u^{\gamma}\right)=f\left(v^{-1}, u v\right) .
$$

Another automorphism ८ of $G$ is defined by

$$
u^{\imath}=v, \quad v^{\imath}=u, \quad z^{\imath}=z^{-1}
$$

and on applying a similar argument it follows that $f(v, u)^{-1}=f(u, v)$.

Finally it is a consequence of all these equations and (ii) that

$$
\begin{aligned}
\left(v^{\phi}, u^{\phi}\right) & =v^{\phi} u^{\phi}\left(v^{\phi}\right)^{-1}\left(u^{\phi}\right)^{-1}=v^{\phi} u^{\phi}\left(v^{-1}\right)^{\phi}\left(u^{-1}\right)^{\phi} \\
& =f(u, v)^{\phi}\left(f\left(v^{-1}, v u\right)^{\phi} f\left(u^{-1}, v u v^{-1}\right)^{\phi}(v, u)^{\phi}\right. \\
& =f(u, v)^{\phi} f\left(v^{-1}, u v\right)^{\phi} f\left(u^{-1}, u\right)^{\phi}(v, u)^{\phi} \\
& =f(u, v)^{\phi} f(v, u)^{-1 \phi}(v, u)^{\phi}=\left(f(u, v)^{2}(v, u)\right)^{\phi} ;
\end{aligned}
$$

and this last expression is not equal to one, since $(v, u)=z^{-1}$ is not the square of an element in $Z$. Thus $H$ is not an abelian group.

If $Z$ is a cyclic group of order $2^{n}$ for $0<n, z$ an element generating $Z$, if $G^{*}$ is a direct product of two cyclic groups of order $2^{n+1}$, and if $u^{*}, v^{*}$ is a basis of $G^{*}$, then let $G$ be the group which satisfies $C(G)=Z \leqq Z(G), G / Z=G^{*}$, and which realizes the operations characterized by $u^{*} \circ v^{*}=z, P\left(2^{n+1}, u^{*}\right)$ $=P\left(2^{n+1}, v^{*}\right)=1$. (This group $G$ has been discussed at the end of $\$ 8$.) $G$ is conformal to an abelian group, but practically the same argument as the one used above proves that there does not exist a transformation $\phi$ into an abelian group which satisfies the conditions (i) to (iv).

COROLLARY B.3. If $G$ is a group with abelian central quotient group, and if $C(G)=C(G)^{2}$ does not contain elements of order 2 , then there exists a conformality $\phi$ of $G$ upon an abelian group $H$ which satisfies the conditions (1) and (2) of Theorem B.1 and transforms the (proper or improper) automorphisms of $G$ into automorphisms of $H$. 
This is a consequence of the fact that every automorphism of $G$ maps $C(G)$ upon a subgroup of $C(G)$ and of Theorem B.1.

Note that the conditions of this Corollary B.3 are satisfied, if $G / Z(G)$ is an abelian group whose elements are of finite odd order.

Appendix C. Operator groups. If $G$ is a group, then $\Phi$ is said to be a set of operators for $G$, if $x^{\phi}$ is, for every $x$ in $G$ and for every $\phi$ in $\Phi$, a uniquely determined element in $G$ such that $(x y)^{\phi}=x^{\phi} y^{\phi}$. $\Phi$ may be called an associative set of operators for the group $G$, if a multiplication of the elements in $\Phi$ is defined which satisfies $\left(x^{\phi}\right)^{\gamma}=x^{\phi \gamma}$.

If $G$ is a group, and if $\Phi$ is a set of operators for the group $G$, then the subgroup $S$ of $G$ is said to be $\Phi$-admissible if $S^{\phi} \leqq S$ for every $\phi$ in $\Phi$. The commutator group $C(G)$ is $\Phi$-admissible for every set $\Phi$ of operators. But it is easy to construct groups $G$ and sets $\Phi$ of operators of $G$ such that the central $Z(G)$ is not $\Phi$-admissible.

If $G$ is a group, and if $\Phi$ is a set of operators for $G$, then the $\Phi$-central $Z(G, \Phi)$ of $G$ may be defined as the set of all those elements $x$ in $G$ which satisfy $x y=y x$, for every $y$ in $G$, and $x^{\phi}=x$, for every $\phi$ in $F$; and the subgroup $C(G, \Phi)$ of $G$, which is generated by the elements $(x, y)$ and $x^{\phi} x^{-1}$ for $x, y$ in $G$ and $\phi$ in $\Phi$, may be called the $\Phi$-commutator group of $G$. Both $Z(G, \Phi)$ and $C(G, \Phi)$ are normal $\Phi$-admissible subgroups of $G . Z(G, \Phi)$ is the greatest subgroup of $G$ in which the inner automorphisms of $G$ and the operators in $\Phi$ induce the identity transformation. $C(G, \Phi)$ is the smallest subgroup of $G$ whose quotient group is abelian and in whose quotient group all the operators in $\Phi$ induce the identity only. Thus $Z(G, \Phi)$ and $C(G, \Phi)$ are $\Phi$-characteristic subgroups of the $\Phi$-operator group $G$.

The obvious generalization of the groups with abelian central quotient group are the groups satisfying the equation

$$
C(G, \Phi) \leqq Z(G, \Phi) \text {. }
$$

They are groups with abelian central quotient group, since $C(G) \leqq C(G, \Phi)$ and $Z(G, \Phi) \leqq Z(G)$. Thus it is advisable to consider $G$ as an extension of a group $S$ between $C(G, \Phi)$ and $Z(G, \Phi)$ ( $S$ is then also situated between $C(G)$ and $Z(G)$ ) by the group $G / S=G^{*} . S$ and $G^{*}$ are abelian groups essentially without operators. Hence the only problem is to characterize the operators in $\Phi$ by relations between $G^{*}$ and $S$, and this may be done by practically the same method as has been used in $\$ 10$ in order to describe the automorphisms in the group $\Omega(S<G)$ since the elements in $\Phi$ induce automorphisms of $G$ which belong (as automorphisms) to $\Omega(S<G)$.

The University of Illinois,

URBANA, ILl. 